\title{
Investigation of the effects of quarl and initial conditions on swirling non-premixed methane flames:
}

\section{Flow field, temperature, and species distributions}

\author{
A.M. Elbaz ${ }^{1,2}$ and W.L. Roberts ${ }^{1}$ \\ ${ }^{1}$ Clean Combustion Research Center, King Abdullah University of Science and Technology, KAUST, \\ Saudi Arabia. \\ ${ }^{2}$ Faculty of Engineering-Mattria, Helwan University, Cairo, Egypt.
}

\section{Abstract}

Detailed measurements are presented of the turbulent flow field, gas species concentrations and temperature field in a non-premixed methane swirl flame. Attention is given to the effect of the quarl geometry on the flame structure and emission characteristics due to its importance in gas turbine and industrial burner applications. Two different quarls were fitted to the burner exit, one a straight quarl and the other a diverging quarl of $15^{\circ}$ half cone angle. Stereoscopic Particle Image Velocimetry (SPIV) was applied to obtain the three components of the instantaneous velocity on a vertical plane immediately downstream of the quarl exit. Temperature and gaseous species measurements were made both inside and downstream of the quarls, using a fine wire thermocouple and sampling probe respectively. This work provides experimental verification by complementary techniques that, although the main flame structures were governed by the swirl motion imparted to the air stream, the quarl geometry, fuel loading and air loading also had a significant effect on the flow pattern, turbulence intensity, mixture formation, temperature distribution, emissions and flame stabilization. Particularly, in the case of the straight quarl flame, the flow pattern leads to strong, rapid mixing and reduces the residence time for NO formation within the internal recirculation zone IRZ. However, for the diverging quarl flames, the recirculation zone is shifted radially outward, the turbulent interaction between the central fuel jet and the internal recirculation zone IRZ induces another small vortex between these two flow features. Less mixing near the diverging quarl exit is observed, with a higher concentration 
of NO and $\mathrm{CO}$ in the post-combustion zone. The instantaneous flow field for both flames showed

29 the existence of small scale vortical structure near the shear layers which were not apparent in the time averaged flow field. These structures, along with high levels of turbulence present in the

31 shear layer, result in the enhancement of the mixing, especially with straight quarl. The main

32 goals of this article are to outline results that yield deeper insight into the combustion of swirling

33 flames stabilized within the quarl and to establish an experimental data base for modelers.

\section{1. Introduction}

Swirl flows have been widely investigated because of their extensive use in a wide range of practical combustion systems, including gas turbine combustion, industrial burners, and furnaces

37 [1-2]. Although a quarl is commonly used in gas turbine and industrial burners with swirling 38 flames, there have been few studies on the effect of the qural geometry on the combustion 39 characteristics. Many free or confined swirl diffusion flame studies have been performed using 40 burners with a straight exit burner (i.e., no quarl). Experimental results have established the 41 general characteristics of swirl flows and revealed important effects of swirl on promoting flame 42 stability and increasing combustion efficiency [3-7].

43 Measurements of the concentrations of gas species, temperature and emissions of pollutants 44 in non-premixed swirling flames are presented in [8-10]. Two typical flame configurations arise 45 according to the primary air ratio in non-premixed propane swirling flames. In the first type of 46 flame, the fuel burns at near stoichiometric conditions to form a ring flame around the 47 recirculation zone. In the second type, the fuel layer is diluted by mixing with surrounding air. 48 The second flame type showed a lower $\mathrm{NO}_{\mathrm{x}}$ emission relative to the first one but with a higher concentration of unburned hydrocarbons [8]. The effect of fuel-air mixing on the flame structure 50 and $\mathrm{NO}_{\mathrm{x}}$ emissions in swirling methane jet flames was experimentally studied [9]. Two modes of 
51 combustion effected the emissions: type 1 was fuel jet dominated combustion and type 2 was

52 strongly recirculating combustion. In strongly recirculating flames, a uniform mixture is formed

53 in the recirculation zone that helps to reduce the $\mathrm{NO}_{\mathrm{x}}$ emissions relative to the fuel jet dominated

54 combustion mode. The $\mathrm{NO}_{\mathrm{x}}$ emission level can further be reduced by using an annular fuel

55 injector with a significant decrease in $\mathrm{CO}$ concentration due to enhanced mixing. In-flame

56 measurements of flow field, temperature, and gas concentration were conducted to clarify the

57 mechanism of NO formation [10] in these two types of combustion.

58 Laser-based diagnostic techniques offer the ability to yield two-dimensional fields of velocity

59 and scalars with high temporal and spatial resolution. These techniques were employed in [11] to

60 measure the flow field, structure, temperature, and species distributions of a swirl flame in a

61 laboratory-scale gas turbine. Three flames with thermal powers between 7.6 and $34.9 \mathrm{KW}$ were

62 investigated. These flames were observed to behave differently with respect to combustion

63 instabilities. In addition, the near-field flow structure of isothermal swirling flows and reacting

64 non-premixed swirling flames was measured in a straight exit laboratory burner without quarl by

65 [12]. Two fuel injection geometries, co-axial and radial, leading to different mixing mechanisms

66 were investigated and characterized. Their study indicated that, although the main flame

67 structure and mixing process are governed by the swirl motion, the fuel injectors play an

68 important role in flame stabilization. Simultaneous planar laser induced fluorscense (PLIF) on

$69 \mathrm{OH}$ radical and particle image velocimetry (PIV) measurements in a gas turbine model

70 combustor were used to investigate the influence of turbulence on local flame characteristics

71 [13]. These measurements revealed the formation of reaction zones in regions where hot burned

72 gas from the recirculation zones mixed with the fresh fuel/air mixture at the nozzle exit. 
74 examined by Presser et al. [14]. Under non-burning and burning conditions, the fuel-air

75 interaction in a kerosene spray swirling flames was examined, and provided qualitative details

76 and quantitative measurements of the induced spray. The effects of the physical properties of

77 four different fuels on the swirl spray flames was addressed by these researchers in [15]. They

78 concluded that the fuel viscosity effected the mean droplet size and spray velocity distribution,

79 with a negligible effect of the surface tension. The flame structures were seen to be influenced by

80 the fuel volatility and with increasing carbon to hydrogen fuel ratio, the flame luminosity was

81 observed to increase. Straight exit, double swirl burners have been proposed as a method to

82 develop air staging to reduce NOx burner [16-17]. They showed that the radial distribution of

83 species and temperature differs significantly in the air staged flames, for which some

84 interpretations have been suggested. The most important parameter was the air distribution,

85 which can lead to air staging along the flame length and reductions of NOx to one third of that

86 the flames without staging [16]. The details of the swirl effects on the combustion characteristics

87 of premixed swirling flames issued from a double concentric swirl burner was examined by

88 Gupta et al. [18] and Marshall and Gupta [19]. The temperature (mean and fluctuations) field of

89 two co-swirl and counter-swirl flames has been investigated. The symmetry of the flame and

90 NOx emissions were found to be greatly influenced by the swirl direction. Most of these studies

91 investigated swirling flames with a straight exit into a free environment [8, 9, 12, 14-19] and/or

92 in a gas turbine model combustor $[11,13]$.

93 Relevant swirling flame work with a quarl, includes a study of the stability maps of a swirl-

94 stabilized, nonpremixed natural gas burner with three quarls and three fuel nozzle arrangements

95 by Milosavljevic et al. [20]. The quarl half-angles were $20^{\circ}$ and $25^{\circ}$, the ratios of length to 
through diameter were $\mathrm{L} / \mathrm{D}=1$ and 1.9 , and the maximum swirl number was 1.8 . They

97 concluded that the leanest flames were found in the $20^{\circ}, \mathrm{L} / \mathrm{D}=1$ quarl, and the poorest stability was provided by the $25^{\circ}, \mathrm{L} / \mathrm{D}=1.9$ quarl. In a different study, the flow field and flame structure

99 of a turbulent non-premixed swirl flame with a straight exit burner (no quarl) was measured by Shen et al. [21] and results were compared with existing data on a diverging quarl flame in [22] to provide further insight into the effect of the burner geometry on the flame structure. The flow field of their study was determined using the isothermal equivalent to the swirling flames via a laser Doppler velocimeter.

104 It is clear that the effects of a quarl on the flow field and combustion of reacting swirling 105 measurements are important for establishing real boundary conditions. Here, the influence of the initial conditions (both fuel and air loading) on the structure of a free turbulent swirl diffusion flame with either a straight or diverging quarl is experimentally investigated. Comprehensive measurements of turbulent near flow field and just above the quarl exit using stereoscopic PIV and in-flame measurements of temperature and chemical species concentration inside of and 112 downstream from the quarl are reported.

\section{3}

114

115 116

117

\section{Experimental setup and measurement techniques}

A schematic of the swirl-stabilized flame apparatus is shown in Fig. 1a. The burner consisted of two concentric tubes with an annulus that supplied swirled air and a central pipe delivering the fuel (methane). The swirl was generated via four tangential air inlets that mixed the tangential air with axial air upstream of the burner. The swirling coaxial airflow surrounded the central fuel tube with an inner diameter, $\mathrm{d}_{\mathrm{f}}$, of $4.4 \mathrm{~mm}$ and outer diameter, $\mathrm{d}_{\mathrm{fo}}$, of $6.3 \mathrm{~mm}$. The air tube 
119 diameter, $\mathrm{d}_{\mathrm{A}}$, at the throat $(\mathrm{Z}=0 \mathrm{~mm})$ indicated in Fig.1a, was $27 \mathrm{~mm}$. The flame was 120 established in a quarl downstream from the exit of the air tube. Two different quarls were fixed 121 at the exit of the burner, namely a straight quarl (shown in Fig. 1a) and a diverging quarl with a 122 half cone angle of $15^{\circ}$ as shown in Fig. 1b. The length, L, of both quarls was the same, $40 \mathrm{~mm}$. 123 This burner was specifically designed to facilitate accessibility to radial and axial measuring 124 probes inside the quarl for gas species concentration and temperature measurements. These 125 measurements were done inside the quarl via four vertically aligned holes that were $3 \mathrm{~mm}$ in 126 diameter on one side of the quarl. Only one measuring hole was used at a time with the other 127 three holes sealed, such that no air could be entrained into the upstream region of the flame 128 through these holes.

129 As shown in Fig. 1c, the stereoscopic PIV system consisted of a dual cavity, diode-pumped, 130 solid-state Nd:YLF laser (LDY 300 Series) and a pair of CMOS cameras (LaVision, Image Pro 131 HS 4M, HSS5, with a maximum resolution of $2016 \times 2016$ pixel). The laser produced $3.5 \mathrm{~mJ} /$ 132 pulse at a repetition rate up to $10 \mathrm{kHz}$, with $9 \mathrm{~ns}$ pulse duration. Pulses were formed into a sheet 133 with a $0.5-\mathrm{mm}$ waist along the flame centerline using three cylindrical lenses. Both the air and 134 fuel jets were seeded with titanium dioxide $\left(\mathrm{TiO}_{2}\right)$ particles with a nominal diameter of $0.5 \mu \mathrm{m}$ 135 via two seeding units (Particle Blaster 100- LaVision). The PB 100 seeding unit consists of a 136 cylinder with a variable speed magnetic stirrer and plate with six nozzles to introduce the carrier 137 flow inside the cylinder (the cylinder is partially filled with the seeding powder). The magnetic 138 stirring bar inside the housing actuates the particles; by the changing the speed of rotation, 139 agglomeration of the seeding particles could be prevented. In addition, the seeding unit 140 incorporates a heating unit to remove any humidity from the seeding powder. The cut-off 141 frequency in the turbulent flow field of the seeding particles is estimated to be $60 \mathrm{KHz}$, based on 
142 the interpolation formulae: $\mathrm{f}_{\text {cut-off }} \approx \nu / \pi\left(\varepsilon_{\text {cut-off }} / a\right)^{2}$ as suggested by Mei [23], where a is the seeding 143 particle radius, $v$ is the kinematic viscosity, and $\varepsilon_{\text {cut-off }}$ is a function in the density ratio $\rho$, defined 144 as the ratio between the seeding particle and fluid density and estimated as: $\left(\varepsilon_{\text {cut-off }} \approx\left[\left(\left(3 / 2(\rho)^{\wedge} 0.5\right)^{\gamma}\right.\right.\right.$ $\left.145+\left(0.932 /(\rho-1.621)^{\gamma}\right]^{1 / \gamma}\right)$. The cameras were mounted equidistant from the centerline of the burner 146 with an angle of separation of $35^{\circ}$. Mie-scattered light from the particle-laden flow was collected 147 using a $105 \mathrm{~mm}, \mathrm{f} / 4$ objective lens (Nikon UV Micro-Nikkor) equipped with a $527 \mathrm{~nm}$ band pass 148 filter. The cameras were operated in two-frame burst mode at $500 \mathrm{fps}$. Image-blur due to off149 axis defocusing was corrected using Scheimpflug adaptors between the objectives and the cameras. Perspective distortion was corrected using a dual plane, three-dimensional imaging 151 target (LaVision Type 22). Image de-warping between the two camera images was corrected 152 using the image correction and distortion function. Also, with the self-calibration function, the 153 coordinate system and the camera calibration of this stereoscopic PIV setup were adjusted so that 154 the $\mathrm{Y}=0 \mathrm{~mm}$ plane (see Fig. 1a. for coordinates) was adjusted exactly in the middle of the laser 155 light sheet. Vector fields were computed from particle image spatial cross correlations using the 156 La Vision Davis 8.1 software package. An adaptive multi-pass vector evaluation technique was 157 used, with interrogation boxes ranging from 128 pixels to 16 pixels, with $50 \%$ overlap resulting 158 in spatial resolution and vector spacing of approximately $0.5 \times 0.5 \mathrm{~mm}$ and $0.25 \mathrm{~mm}$, 159 respectively. The final velocity vector fields were smoothed with a $3 \times 3$ vector moving average 160 filter for subsequent analysis.

161 A 3-D positioning mechanism was used to move the measuring probes for temperature and 162 gas concentration measurements in all three spatial dimensions with a resolution of $0.1 \mathrm{~mm}$. The 163 temperature distribution of the flame was measured using a $12-\mu \mathrm{m}$ wire diameter uncoated type$164 \mathrm{~S}(\mathrm{Pt}-\mathrm{Pt}-13 \% \mathrm{Rh})$ thermocouple. The thermocouple (TC) signal was digitized and recorded by a 
165 PC using an AD Lab Card (model NI PCI-6020, 16-bit, maximum sampling rate $200 \mathrm{KHz}$ ). Due 166 to the relatively small wire diameter of the thermocouple, the temperature measurements in this 167 work were uncorrected for radiation [24]. The maximum error in temperature measurements, 168 neglecting the influence of radiation, was estimated to be $\pm 50 \mathrm{~K}$. A water-cooled sampling 169 probe with an inner diameter of $1 \mathrm{~mm}$ and a $2.5 \mathrm{~mm}$-diameter outer cooling tube were used to 170 collect gas samples for both in-flame and post-combustion measurments. The tip of the outer 171 cooling tube was tapered to an inner diameter of $1 \mathrm{~mm}$, such that the disturbance to the flow was 172 minimized. The gas sample was cooled down to $60{ }^{\circ} \mathrm{C}$ to yield water vapor condensation and the 173 concentrations of $\mathrm{O}_{2}, \mathrm{CO}$ and $\mathrm{NO}$ were measured using a LANCOM series II gas analyzer. The 174 uncertainty in the gas species concentrations were $\pm 0.1 \%$ in $\mathrm{O}_{2}, \pm 1 \mathrm{ppm}$ for $\mathrm{NO}$, and $\pm 50 \mathrm{ppm}$ in 175 CO concentrations.

176 The swirl number in this study is defined as the geometrical swirl number and calculated by 177 measuring the mass flow rates of the axial air $\left(\mathrm{m}_{\mathrm{A}}\right)$ and tangential air $\left(\mathrm{m}_{\theta}\right)$, which is defined as $178 \quad[25]:$

$$
S_{g}=\frac{\pi r_{o d_{A}}}{2 A_{t}}\left(\frac{m_{\theta}}{m_{\theta}+m_{A}}\right)^{2}
$$

where $S_{g}$ is the geometric swirl number, $A_{t}$ is the total area of the four tangential air inlets, with $181 \mathrm{~d}_{\mathrm{t}}=4.4 \mathrm{~mm}, \mathrm{r}_{\mathrm{o}}$ is the radius of the air tube $\left(\mathrm{d}_{\mathrm{A}}-\mathrm{d}_{\mathrm{t}}\right)$, and $\mathrm{d}_{\mathrm{A}}$ is the inner diameter of the air tube, $182 \mathrm{~d}_{\mathrm{A}}=27.2 \mathrm{~mm}$. In this work, the swirl number was 10.25 .

\section{3. Results and Discussion}

185 3.1. Flame stability and appearance 
With a fixed geometrical swirl number, $\mathrm{Sg}$, of 10.25 , the flame stability limits were measured with two quarls, a straight and diverging quarl, fitted to the burner exit. These stability results are presented as the relation between the axial air velocity, $\mathrm{Va}$ (calculated based on the axial flow rate and the annulus area), and the fuel jet velocity at blow off, $\mathrm{Vf}_{\text {blow-off. }}$ The stability point was reached by gradually reducing the fuel flow rate while keeping the axial and tangential air flow rates constant until the flame was extinguished. As illustrated in Fig. 2, the fuel velocity at extinction, $\mathrm{Vf}_{\text {blow-off }}$, generally increases with increasing axial air velocity. The flames with the straight quarl were more stable than those with the diverging quarl, and this effect was more 194 pronounced at high axial air velocities. The possible physical mechanism leading to this difference is the zero axial velocity contour in the straight quarl, where the strain rates are relatively low, substantially improving the stability limits. This hypothesized mechanism is confirmed by the in-flame measurements.

By decreasing the fuel jet velocity in the straight quarl, the effect of the internal recirculation zone, IRZ, through the backflow becomes dominant, which moves the forward stagnation point further upstream towards the fuel tube. This leads to more fuel/air mixing with hot combustion 201 products continuously mixed with the fresh fuel/ air mixture and to a higher probability that the 202 instantaneous flammable region overlaps the zero axial velocity contours. Contrary to this, in the 203 diverging quarl, the IRZ is shifted radially away from the fuel jet, which means that there is less 204 of an interaction between the fuel jet and the vortex in the early axial zone of the flame. With this 205 shifted IRZ, less mixing and more unheated recirculated gases are pushed toward the fuel jet 206 shear layer. Therefore, when the flame approaches extinction by decreasing the fuel jet velocity, 207 which increases the percentage of unheated recirculated gases relative to the fuel jet, which in 208 turn, leads to the sudden blow off of the flame in the diverging qual. 
Based on the stability plot, in-flame measurements were conducted on five selected flames.

210 The main parameters of these selected flames and the corresponding flame designations are

211 listed in Table 1, and their locations on the stability curves are shown in Fig. 2. Two sets of 212 experiments with a straight quarl were conducted. In the first set, the axial air velocity was $2.7,4$, 213 or $6 \mathrm{~m} / \mathrm{s}$, while the jet fuel velocity, $\mathrm{V}_{\mathrm{f}}$, was kept constant at $5.8 \mathrm{~m} / \mathrm{s}$. In the second set, with a 214 constant axial air velocity, $\mathrm{V}_{\mathrm{a}}$, of $4 \mathrm{~m} / \mathrm{s}$, the fuel jet velocity was $3.5,5.8$, or $7.3 \mathrm{~m} / \mathrm{s}$. One flame 215 condition $\left(\mathrm{V}_{\mathrm{f}}=5.8 \mathrm{~m} / \mathrm{s}\right.$ and $\left.\mathrm{V}_{\mathrm{a}}=4 \mathrm{~m} / \mathrm{s}\right)$ with the diverging quarl and same swirl number, was 216 investigated. As indicated in Table 1, among the five conditions in the flame appearance 217 investigation, four flames were selected for Stereo PIV and temperature/gas species 218 measurements.

219 Photographs with exposure time of $1 / 30 \mathrm{~s}$ of the swirling methane flames with $\mathrm{Sg}=10.25$ for the 220 three different $\mathrm{V}_{\mathrm{a}}$ of $2.7,4$, and $6 \mathrm{~m} / \mathrm{s}$ are shown in the first row of Fig. 3. At low axial velocity 221 (left photo), a blue bubble-like recirculation zone appeared at the flame base at the quarl exit. 222 The flame was long and its highly luminous yellow jet flame fully penetrated the recirculation 223 zone, indicating insufficient mixing. Increasing the axial air velocity to $4 \mathrm{~m} / \mathrm{s}$ shortened the flame 224 length and a more compact blue flame was formed, indicating the formation of a strong internal 225 recirculation zone that enhanced fuel-air mixing. As the axial air velocity was further increased 226 (third photo), the flame became blue, indicating high and uniform temperature distribution. 227 Moreover, the flame was acoustically noisy, resulting from the violent interaction of the central 228 jet and recirculation vortex and/or vortex breakdown. The first three photos of the second row 229 show the effect of increasing the fuel jet velocity on flame appearance when $\mathrm{Sg}$ and axial 230 velocity were kept constant (see Table 1). With increasing fuel velocity, the internal recirculation 231 zone, IRZ, was seen to intermittently penetrate the flame and a yellow plume appeared (Flame 
232 Fs5) and the yellow plume appears as a result of penetration of the fuel jet to the IRZ and 233 increases as the fuel jet increases. This luminosity indicated the formation of soot that was 234 confined to the central region, likely due to the intermittent formation of a large concentration of 235 fuel in the high temperature regions of the flame, see flames Fs1 and Fs5. Also, as shown in the 236 last photo in the second row, the diverging quarl had a significant effect on the flame appearance 237 (flame Fd2). In this case, the diverging quarl led to the fuel being able penetrate through the IRZ, 238 which was not the case with the straight quarl under the same operating conditions (flame Fs2). 239 With a diverging quarl, the IRZ shifted radially outward, which reduced the momentum exerted 240 from the reverse flow on the central fuel jet at locations low in the flame.

\subsection{Flow field features}

242 Figure 4 shows ensemble averaged images of 500 stereo PIV recordings for swirling flames

243 Fs2, Fs3, Fs4, and Fd2. Only half of the PIV measurements are presented due to symmetry.

244 These images were recorded over the entire region of the flame above the quarl exit. The flow 245 swirled counter-clockwise about the vertical axis (as viewed from above) and the highest flow 246 velocities were seen in the conically shaped inflow of fresh gases. The velocity vector values in

247 the measurement plane are indicated by the arrows and are superimposed with streamlines; the 248 out-of-plane component (W) is discussed in the next section. Typical features of the flow field of 249 a swirling flame accompanied by a straight quarl (Fs2) are shown in Fig. 4a. The flow field is 250 characterized by an inner recirculation zone, IRZ. The eye of this IRZ appears immediately at the 251 quarl exit $(-15<\mathrm{r}<-5 \mathrm{~mm})$, and, as indicated, the IRZ extends down inside the quarl. The 252 negative axial velocity in the near field around the flame's central region indicates that the 253 backflow and the fuel jet formed a stagnation point inside the quarl. This IRZ is flanked by a 254 velocity peak (at nearly $\mathrm{r}=-16 \mathrm{~mm}$ ) associated with the faster flow through the annular swirling 
255 jet. A shear layer exists between the inflow swirling jet and IRZ. In this shear layer, hot 256 combustion products are continuously mixed with the fresh fuel-air mixture, and combustion is 257 stabilized such that the flame resides here. Further downstream, the inward flow toward the 258 central region disappears. As shown in Fig. 4b, increasing the axial air velocity leads to more 259 outward radial expansion of the swirling jet. This toroidal recirculation zone increases with axial 260 air velocity. Moreover, the radial expansion and the higher axial velocity leads to the 261 downstream movement of the center of the IRZ, while its radial location remains nearly fixed. 262 Contrary to this behavior, decreasing the fuel jet velocity of flame Fs3 (Fig. 4c) causes the center 263 of the IRZ to move upstream, closer to the quarl exit.

264 The flow field for the diverging quarl (Fd2, Fig. 4d) exhibits different flow field features 265 compared with those of flame Fs2. In the near field close to the quarl exit, three different flow 266 regions are observed: a positive velocity vector around the flame's centerline due to the fuel jet; 267 an outer recirculation zone located far from the flame's centerline; and an inner vortex generated 268 between the fuel jet and this outer recirculation zone. This inner vortex is generated at the shear 269 layers of these two large features and this inner vortex is responsible for the mixing between the 270 fuel jet and the main swirling jet at locations near the fuel tube's exit. With the diverging quarl, 271 the swirling jet expands radially, leading to the creation of an inner vortex far away from the 272 flame's centerline (see Fig. 4-d). This in turn allows the central fuel jet to penetrate through the 273 recirculation backflow, leading to less mixing between the fuel and air and resulting in soot 274 formation within the central region of that flame that was observed in the flame appearance. 275 Figures (4e-4f) illustrate the average velocity vectors superimposed the strain rate $(\partial \mathrm{v} / \partial \mathrm{z}$, where $276 \mathrm{~V}$ is the vertical velocity component and $\mathrm{z}$ is the vertical axial distance) at the lower part of 277 flames Fs2 and Fd2. As shown close to the burner exit, the zero velocity line of the IRZ for 
278 flame Fs2 is localized within a region of relatively low strain rate. However, in the flame Fd2,

279 the recirculation zone is shifted radially, with the flame residing in a region of relatively high

280 strain rate where the flame is reside there.

281 While the ensemble-averaged flow field provides insights into the flame's stabilization 282 mechanisms, instantaneous flow structures play a more critical role in determining combustion 283 stability, mixing enhancement, and flame blow off. Instantaneous structures of the flow field are 284 shown in Fig. 5 with two examples of the velocity vectors and a streamline plot from a time 285 series of the straight and diverging quarls under F2 conditions. It is immediately clear that the 286 instantaneous flow field looks dramatically different from the mean flow field and that strong 287 temporal fluctuations of the structures occur. In contrast to only a single internal recirculation 288 zone observed in the mean velocity field, the instantaneous PIV images indicate the existence of 289 multiple small-scale vortical structures in regions close to the shear layers and at further axial 290 distances from the central region of the flame, see Fig. 5 which shows two examples of 291 consecutive flow field plots at $500 \mathrm{fps}$. These small vortices form and dissipate continuously in 292 the shear layer. Along with the high turbulence intensity present in the shear layer, these vortices 293 result in intense mixing between the cold fresh gas and hot burned gases coming from IRZ in 294 flame Fs2. However in flame Fd2, the intensity of the mixing is reduced due to the radial 295 expansion of the swirling jet flow. Figures $5 c-5 d$ illustrate the mutual interaction between the 296 central fuel jet and the swirling main jet in the shear layers of the inner vortex. In addition, in 297 both flames, the instantaneous velocity fields are much larger than those in the ensemble 298 averaged fields shown in Fig. 4. Furthermore, at some locations, sudden increases in velocity 299 occur within the instantaneous field of flow, and these increases are averaged out in the mean 300 flow field. According to mass conservation requirements, strong swirling flow components 
normal to the measurement plane are needed to support such increases or decrease. Fig. 5e shows an example of these increases, where the planner velocity vectors are overlaid with the swirling velocity in a typical instantaneous velocity image. The locations where the velocity suddenly increases are associated with a lower tangential velocity and vice versa.

The centerline profiles of the mean axial velocity, $\mathrm{V}$, and the central turbulence intensity 306 characterized by the root mean squared rms of the axial velocity, Vrms, are shown in Fig. 6. As 307 illustrated in Fig. 6a, the three flames with the straight quarl all show a negative centerline 308 velocity across the IRZ. Further downstream, these profiles show a progressive velocity increase 309 until reaching the boundary of the IRZ. Downstream of the IRZ, the centerline velocity profiles 310 show a nearly linear centerline increase, with the highest centerline velocity for flame Fs2 and 311 the lowest centerline velocity for flame Fs4, due to the lower thermal input of flame Fs4. The 312 corresponding mean centerline temperature distributions for these flames are shown in Fig. 7. 313 The data indicate that there is a gradual centerline temperature increase with the diverging quarl 314 compared to a steep temperature increase with the straight quarl. Beyond the peak temperature, 315 flame Fs4 experiences a linear decline in temperature; flame Fs3 experiences a significant 316 decrease in temperature around the boundaries of IRZ. However in the near field, due to the 317 penetration of the fuel jet into the IRZ (Fig. 6a.), the centerline velocity of flame Fd2 experiences 318 a gradual decrease, reaching a minimum value at $\mathrm{Z}=80 \mathrm{~mm}$. This decrease is due the fuel jet319 vortex interaction. Downstream of this location, the centerline velocity recovers, exhibiting a 320 higher centerline velocity in comparison with Fs2.

321 Two factors lead to this behavior: the first is a reduced effect from the IRZ on the early fuel 322 jet for flame Fd2, and the second is the delay of mixing, which increases the flame's centerline 323 temperature in the post-combustion flame region (see Fig. 7). Axial profiles of the vertical 
324 velocity rms, Vrms, are shown in Fig. 6b. The data show similar profiles for all flames with

325 straight quarls, where Vrms is lower in the near field with a steep increase, reaching a peak at the

326 boundary of the IRZ. For flames with diverging quarls, a remarkably high and constant Vrms at

327 the quarl exit exists for a short distance (attributed to the central fuel jet) and is followed by a

328 steep increase in Vrms, reaching a peak at the axial location of minimum V. In general, the Vrms

329 of the diverging quarl exhibits higher velocity fluctuations relative to the flames with the straight

330 quarl due to the vigorous fuel jet-vortex interaction downstream of the quarl exit plane. In

331 addition, the early mixing of straight quarl flames leads to regions of higher temperature regions

332 in the lower parts of flame, and hence more damping of the velocity fluctuations.

333 The mean swirling velocity component, W, was investigated by the ensemble average of 500

334 stereo PIV recordings of flames Fs2 and Fd2, with the contours of the mean swirling velocity

335 shown in Fig. 8. One can see that, in both flames, the maximum swirling velocity components

336 are located downstream from the quarl exits. In addition, with the radial expansion of the

337 swirling jet associated with the diverging quarl, these flow conditions conserve the tangential

338 velocity for a longer distance further downstream from the quarl, while pushing the location of

339 the maximum swirling velocity downstream. This may help explain the penetration of the fuel jet

340 into the IRZ with the diverging quarl. The contours shown in Fig. 8 and the radial profiles of the

341 swirling velocity and the swirling velocity fluctuations at $\mathrm{Z}=80 \mathrm{~mm}$ shown in Fig. 9 indicate

342 that over the entire region of both flames, no mean tangential component exists at the flame

343 centerline. However, high tangential velocity fluctuations (Wrms) occupy the flame core, with

344 higher fluctuations measured for $\mathrm{Fd} 2$.

345 Although the average velocity vectors show a negative axial velocity for flame Fs2 and a 346 positive axial velocity for flame Fd2 at low axial distances. For example, at an axial distance of 
$347 \mathrm{Z}=60 \mathrm{~mm}$, the axial velocity histograms (Fig. 10) indicate that both negative and positive values

348 exist. With the large velocity fluctuations at the flame's centerline for flame Fd2, the histogram

349 shows a broader velocity band than that of flame Fs2, with the average shifted toward the

350 positive velocity. These velocity fluctuations give an indication of the motion of the stagnation

351 point for flame Fs2, and the higher velocity fluctuations associated with the central penetrated jet

352 of flame $\mathrm{Fd} 2$.

353

\subsection{Temperature field and gas concentrations}

The mean temperature contours for flames Fs2, Fs3, Fs4 and Fd2 are presented in Fig. 11, where these contours result from measurements inside and downstream from the quarl. With the formation of the IRZ at the quarl exit of flame Fs2, it can been seen in Fig. 11a, the highest temperatures were obtained in the main reaction zone, located at the boundary of the IRZ and extending down inside the quarl. In the IRZ close to the axis, where burnt out gases mainly prevail, low heat loss leads to higher temperatures. Further downstream from the IRZ, a narrow annular tail of peak temperature surrounds a relatively low temperature central flame region. With increasing axial air velocity at the same swirling number of flame Fs3, a large IRZ is formed at the quarl exit, leading to stronger inner backflow and resulting in a broader high temperature zone inside the quarl. With increasing axial velocity, the engulfment of the cold mixture in the central region reduces the temperature level immediately downstream of the IRZ. By decreasing the velocity of the fuel jet (flame Fs4), the overall mean gas temperature across the entire flame is reduced (Fig. 11c). The temperature contours of the diverging quarl shown in Fig. 11d indicate a very different temperature distribution. The peak temperature occurs much further downstream and is confined to a narrow region about the centerline, in line with the flow field pattern of the diverging quarl flame. An example of the radial temperature distribution at an 
370 axial distance of $\mathrm{Z}=38$ and $50 \mathrm{~mm}$ (inside and outside the quarl) is shown in Fig. 12a, where the

371 peak temperature for flame Fs2 occurs at the flame centerline compared to radially shifted

372 temperature peak for flame $\mathrm{Fd} 2$ at an axial distance of $\mathrm{Z}=38 \mathrm{~mm}$. With the aid of the radial

373 axial velocity and radial temperature profiles at an axial distance of $\mathrm{Z}=50 \mathrm{~mm}$ for flames Fs2

374 and Fd2, shown in Fig. 12b, the main swirling jet at the diverging quarl is shifted outward. In

375 addition, it is clear that at the fuel jet's shear layer, there is a reversed flow region in flame Fd2

376 and a totally reversed region occupying the central region of flame Fs2. The peak temperature is

377 located in the reversed inner boundaries with the temperature gradually declining toward the

378 main swirling jet in flame Fd2. With the straight quarl, a fairly uniform temperature is observed

379 near the centerline inside the reversed region of flow, with a steeper temperature decline moving

380 radially outward. This indicates that there is less fuel jet swirling in the diverging quarl. In the

381 flow field pattern of the diverging quarl, the fuel jet penetrates the IRZ, and hence a lower

382 temperature is recorded at the flame's centerline inside the quarl, with the formation of the

383 secondary vortex at the shear layer of the fuel jet, leading to a peak temperature located away

384 from the flame's centerline inside the quarl as shown in Fig. 12b. As a consequence, mixing

385 between the fuel and air is postponed to further downstream and a higher temperature is recorded 386 at the flame's tip.

387 Figures 13 and 14 show the radial gas concentrations and mean temperatures in flames Fs2 388 and Fd2 at eight different axial locations. The concentrations are plotted on a dry basis. As 389 shown in Fig. 13, the temperature profile of flame Fs2 inside the quarl and near the burner exit at $390 \mathrm{Z}=28.5 \mathrm{~mm}$ is nearly uniform in the low-temperature region. The concentration measurements 391 indicate a corresponding very low and uniform $\mathrm{CO}$ concentration of $60 \mathrm{ppm}$; the $\mathrm{O}_{2}$ 392 concentration is nearly constant at $20.9 \%$ with a slight decrease at the flame's centerline; and the 
NO concentration is nearly zero. The centerline temperature continuously increases with axial

394 distance, and at $48.5 \mathrm{~mm}$ it reaches $1200{ }^{\circ} \mathrm{C}$, which is accompanied by a steep $\mathrm{CO}$ increase, 395 indicating the mean location of the flame stabilization point. At $63 \mathrm{~mm}$ and further from the fuel 396 tube's exit, the peak temperature is nearly constant and the radial extent of this high temperature 397 region spreads with downstream distance, indicating a well-mixed zone within IRZ. This is 398 coupled with a further reduction in the $\mathrm{O}_{2}$ and $\mathrm{CO}$ concentrations and a progressive increase with 399 a shift in the $\mathrm{CO}$ peak to the boundaries of the uniform flame temperature. Moreover, the NO 400 profiles show a gradual increase as shown in Fig. 13g, they follow the profiles of temperature 401 and the availability of $\mathrm{O}_{2}$ species. The maximum $\mathrm{NO}$ concentration increases with the maximum 402 flame temperature, indicating that the thermal NO mechanism is the dominant mechanism in 403 these regions of the flame.

404 At the furthest downstream locations, $\mathrm{Z}=113$ and $133 \mathrm{~mm}$, the $\mathrm{O}_{2}$ profiles exhibit relative 405 increases within the central flame region, which are attributed to transport of $\mathrm{O}_{2}$ from the outer 406 regions of the flame. The $\mathrm{CO}$ concentration profiles show a gradual reduction in the downstream 407 direction, due to oxidation to $\mathrm{CO}_{2}$. At $\mathrm{Z}=73$ and $83 \mathrm{~mm}$, the $\mathrm{NO}$ concentration peaks at the 408 boundaries of the uniform flame temperature region, but with a more uniform temperature profile 409 at further downstream locations, the NO profiles show an attenuated plateau with the peak 410 displaced away from flame centerline.

411 The radial temperature profiles and concentration profiles for flame Fd2 are shown in Fig.

412 14. The profiles are seen to qualitatively follow the same trends as those of flame Fs2 (Fig. 13). 413 However, due to the diverging quarl, the following features are noted for flame Fd2. The peak 414 flame temperature is located away from the flame's centerline near the exit of the quarl, due to 415 penetration of the fuel jet into the IRZ, and the formation of small vortices at the fuel jet, as 
mentioned earlier. At $\mathrm{Z}=28.5$ and $38.5 \mathrm{~mm}$, a remarkably lower $\mathrm{O}_{2}$ concentration along the

417 centerline is observed followed by a radially sharp increase in the jet shear layer, indicating less

418 mixing in these regions near the quarl exit. Due to the delayed mixing between the fuel jet and

419 swirling air at this early axial distance, the $\mathrm{O}_{2}$ concentration at the centerline is seen to slightly

420 increase downstream, reaching a plateau of uniform $\mathrm{O}_{2}$ concentration, due to the inner vortex

421 located between the fuel jet and the shifted main vortex. At the outer radial boundaries of this

422 plateau, a steep increase in $\mathrm{O}_{2}$ is observed, indicating strong mixing with the outer swirling flow.

423 The sudden increase in CO concentration for flame Fs2 at the boundaries of the IRZ (Fig. 13e) is

424 absent in these flames; rather, a gradual radial increase with the higher concentrations is located

425 at the central flame region. The NO measurements show a sudden increase in the NO 426 concentration between $\mathrm{Z}=38.5$ and $48.5 \mathrm{~mm}$, with a higher concentration at the central region.

427 Based on flame appearance and flow field patterns, we conclude that the diverging quarl 428 leads to insufficient fuel/air mixing at the early axial distances, which leads to a lack of oxygen 429 within the central fuel jet. These conditions indicate that the prompt NO mechanism is the 430 dominant NO formation mechanism at these axial locations near the quarl exit, but further 431 downstream, the NO profiles follow the temperature profile (Fig. 14h) with a higher NO 432 emission with the diverging quarl in comparison with the straight quarl and indicating a switch to 433 the thermal mechanism. Due to back flow in the early regions in flame Fs2, leading to shorter 434 residence times in the recirculation zone and higher recirculated burned gas concentrations, the 435 low NO concentration within IRZ of flame Fs2 is observed.

\section{Conclusions}

437 The design of a quarl-stabilized swirling flame burner for gas turbines and industrial burners 438 requires careful examination of the role of different operational parameters and geometry to 
439 predict flame stability, flame structure, and emissions. The objective of this work was to obtain

440 better insight into the role played by the quarl's geometry on the structure of free-swirling non-

441 premixed $\mathrm{CH}_{4}$ /air flames. The burner was fitted with straight exit or diverging quarl with a $15^{\circ}$

442 half cone angle. With a fixed geometrical swirl number, the effect of axial air velocity, fuel

443 loading, and quarl geometry on the lean stability limit, flame appearance, turbulent flow field,

444 and major gas concentrations inside the quarl and the near field was measured. Due to the lack of

445 optical access inside the quarls, the turbulent flow field was captured only downstream of the

446 quarl exit, using Stereoscopic PIV. However, both the temperature field and major gaseous

447 species concentrations were measured both inside and outside of the straight and diverging

448 quarls.

449 The lean stability limits were strongly influenced by the bulk combustion-air velocity for 450 both quarls, where increasing the axial air velocity reduced the stability flame region, with more 451 broaden flame stability in the case of the straight quarl. The mean flow fields showed that the 452 global mixing process and the main flame structure are governed by the swirling motion exerted 453 on the air stream, and the quarl geometry plays an important role in mixing, flow field features, 454 and flame stabilization. With the straight quarl, the mean flow field can be divided into two main 455 regimes: a conically shaped inflow swirling region and an inner recirculation zone region. The 456 recirculation zone extends down inside the straight quarl. The turbulent interaction between the 457 central fuel jet and the backflow generated within the inner recirculation zone enhanced the 458 mixing just inside the quarl. In contrast, in the diverging quarl flame, the flow field near the quarl 459 exit is characterized by three regions: the central fuel jet region, the outer recirculation region, 460 and between these two regions, the inner recirculation zone. This is accompanied by an outward 461 shift of the conical swirling flow. The inner vortex is responsible for the mixing between the 
462 central fuel jet and the outer recirculation zone. With this flow pattern, less mixing near the quarl 463 exit is observed.

464 The peak swirl velocity is located closer to the straight quarl exit and located farther 465 downstream in the diverging quarl, with a slower rate of decay with axial distance for the 466 diverging quarl. The instantaneous structure of the IRZ consisted of several small vortices 467 mainly located inside the inner shear layer of the swirling jet stream for both quarls. These 468 instantaneous velocity realizations also revealed the existence of small vortices far away from 469 the quarl exit along the flame's central region.

470 Due to different flow field patterns, both the temperature field and species concentrations 471 inside and outside the quarl were affected. In the straight quarl flame, the temperature 472 measurements showed a significant change when both the fuel jet velocity and the axial air 473 velocity were changed. Strong and rapid mixing in these flames increased the mixture's 474 homogeneity and shortened the residence time for NO formation within IRZ, while higher NO 475 and CO concentrations were measured at the IRZ boundaries. In these flames, thermal NO is the 476 dominant formation mechanism.

477 However, the peak flame temperature in the diverging quarl was shifted outward near the 478 quarl exit, where prompt NO is the main mechanism and peak concentrations occurred along the 479 centerline. A steep increase in the $\mathrm{CO}$ concentration at the IRZ boundaries in the straight quarl 480 flame was not observed; rather, a gradual increase with a relatively high concentration in the 481 flame's central region was seen in the diverging quarl. The diverging quarl flames show a higher 482 concentration of $\mathrm{NO}$ and $\mathrm{CO}$ in the post-combustion zone.

483 In conclusion, these experimental measurements provide better understanding of the flow field 484 pattern of swirling flames with quarls and of the effect of this pattern on the flame stability, 
temperature fields, and flame structure in terms of major species. These results will be useful in the validation and improvement of numerical simulations. Future work will focus on further investigations inside the quarl using simultaneous OH PLIF/PIV measurements with optical access via a quartz quarl. With this information, a clearer understanding of the turbulencechemistry interaction in the vicinity of the quarl will be possible. The data will be organized in a usable form and made available, together with information on boundary conditions and estimated measurement accuracies, to model developers.

\section{Acknowledgments}

Research reported in this publication was supported by competitive research funding from King Abdullah University of Science and Technology (KAUST). The temperature field and gas species concentrations measurments were conducted in the Labs of Faculty of EngineeringMateria, Helwan University.

\section{References}

[1] Beér JM, Chigier NA. Combustion Aerodynamics, Applied Science Publishers, London, 1972.

[2] Syred N, Beér JM. Combustion in swirling flows: A review. Combust. Flame. 1974; 23 Issue 2: 143201.

[3] Feikema D, Chen RH, Driscoll JF. Enhancement of flame blowout limits by use of swirl. Combust. Flame 1990; 80: 183-195.

[4] Feikema D, Chen RH, Driscoll JF. Blowout of nonpremixed flames: Maximum coaxial air velocities achievable with and without swirl. Combust. Flame 1991; 86: 347-358.

[5] Meier W, Weigand P, Duan XR, Thoben RG. Detailed characterization of the dynamics of thermoacoustic pulsations in a lean premixed swirl flame. Combust. Flame 2007; 150: 2-26.

[6] Meier W, Duan XR, Weigand P. Investigation of swirl flames in a gas turbine model combustor II. Turbulence-chemistry interactions. Combust. Flame 2006; 144: 225-236.

[7] Faler JH, Leibovich S. Disrupted states of vortex flow and vortex breakdown. Phys. Fluids 1977; 20 : 1385-1400.

[8] Takagi T, Okamoto T. Characteristics of combustion and pollution formation in swirling flames. Combust. Flame 1981; 43: 69-79.

[9] Schmittel P, GÜnther B, Lenze B, Leuckel W, Bockhorn. Turbulent swirling flames: Experimental Investigation of the flow fiels and formation of nitrogen oxide. Proc. Combustion Inst. 2000; 28: 303309.

[10] Cheng TS, Chao YC, Wu DC, Yuan T, Lu CC, Cheng CK, Chang JM. Effects of fuel-air mixing on flame structures and NOx emissions in swirling methane jet flames. 27th Symposium (International) on Combustion 1998; P1229-1237.

[11] Weigand P, Meier W, Duan XR, Stricker W, Aigner M. Investigation of swirl flames in a gas turbine model combustor I. Flow field, structures, temperatures, and species distributions. Combust. Flame 2006; 144: 205-224

[12] Olivani A, Solero G, Cozzi F, Coghe A. Near flow field structure of isothermal swirling flows and reacting non-premixed swirling flames. Experimental Thermal and Fluid science 2007; 31: 427-436.

[13] Sadanandan R, Stöhr M, Meier W. Simultaneous OH-PLIF and PIV measurements in a gas turbine model combustor. Appl. Phys. B 2008; 90: 609-618. 
[14] Presser C, Gupta AK, Semerjain HG. Aerodynamic characteristics of swirling spray flames: pressure-jet atomizer, Coombust. Flame 1993; 92:25-44.

[15] Presser C, Gupta AK, Avedisian CT, Semerjain HG. Fuel property effects on the structure of spray flames, Twenty-Third Symposium (International) on combustion/The combustion Institute, 1990; 1391-1367.

[16] Ballester J, Sanz A, Gonzalez MA, Investigation of the characteristics and stability of air-staged flames, Experimental thermal and fluid science, 2008; 32: 776-790.

[17] Jing J, Li Z, Zhu Q, Chen Z, Wang L, Chen L, influence of the outer secondary air van angle on the gas/particle flow characteristics near the double swirl flow burner, Energy, 2011; Vol 36, Issue 1: 258-267.

[18] Gupta AK, Lewis MJ, Daurer M, Swirl effects on combustion characteristics of premixed flames, Journal of Engineering for gas turbine and power, July 2001; 123: 619-626.

[19] Marshall Aw, Gupta AK. Thermal behavior of coswirling flames, combust. Sci. and Tech.2004; 176: 437-451.

[20] Milosavljevic VD, Taylor AMKP, Whitelaw JH. The influence of burner geometry and flow rates on the stability and symmetry of swirl-stabilized nonpremixed flames. Combust. Flame 1990; 80: 196208

[21] Shen D, Most JM, Joulain P. The effect of initial conditions for swirl turbulent diffusion flame with a straight-exit burner. Combust. Sci. and Tech. 1994; 100, 203-224

[22] Tangirala V, Chen RH, Driscol JF. Effect of heat release and swirl on the recirculation within swirlstabilized flames. Combust. Sci. Technol., 1987; 51, 75-95.

[23] Mei R, Velocity fidelity of flow tracer particles, Experiments in fluids, 1996; 22: 1-13.

[24] Lockwood FC, Moneib HA. Fluctuating temperature measurements in turbulent-jet diffusion flame. Combust. Flame 1982; 47: 291-314.

[25] Claypole TC, Syred N. The effect of swirl burner aerodynamics on NOx formation, Eighteenth Symposium (International) on Combustion, The combustion Institute, Pittsburgh 1981; 18 issue 1: 81- 89. 
494 Table1

495 The main parameters of investigated flames.

\begin{tabular}{|c|c|c|c|c|c|c|c|c|c|}
\hline \multirow{2}{*}{ Quarl } & \multirow[b]{2}{*}{$\mathrm{S}_{\mathrm{g}}$} & \multirow{2}{*}{$\mathrm{Va}(\mathrm{m} / \mathrm{s})$} & \multirow[b]{2}{*}{$\begin{array}{c}\mathrm{Vf} \\
(\mathrm{m} / \mathrm{s})\end{array}$} & \multirow{2}{*}{$\mathrm{mom}_{\mathrm{a}} / \mathrm{mom}_{\mathrm{f}}$} & \multirow{2}{*}{ flame } & \multicolumn{4}{|c|}{ measurements } \\
\hline & & & & & & appearance & SPIV & Temperature & $\begin{array}{c}\text { Gas } \\
\text { concentrations }\end{array}$ \\
\hline \multirow{6}{*}{ 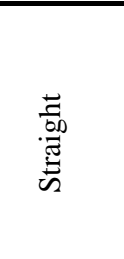 } & \multirow{6}{*}{10.25} & 2.7 & \multirow{3}{*}{5.8} & 13.8 & Fs1 & $*$ & & & \\
\hline & & 4 & & 30.4 & Fs2 & $*$ & $*$ & $*$ & $*$ \\
\hline & & 6 & & 68.4 & Fs3 & $*$ & $*$ & $*$ & \\
\hline & & \multirow{3}{*}{4} & 3.5 & 83.4 & Fs4 & $*$ & $*$ & $*$ & \\
\hline & & & 5.8 & 30.4 & Fs2 & $*$ & $*$ & $*$ & \\
\hline & & & 7.3 & 19.2 & Fs5 & $*$ & & & \\
\hline $\begin{array}{c}\text { Diverging } \\
\theta=15^{\circ}\end{array}$ & 10.25 & 4 & 5.8 & 30.4 & $\mathrm{Fd} 2$ & $*$ & $*$ & * & $*$ \\
\hline
\end{tabular}


498

499

500

501

502

503

504

505

506

507

508

509

510

511

512

513

514

515

516

517

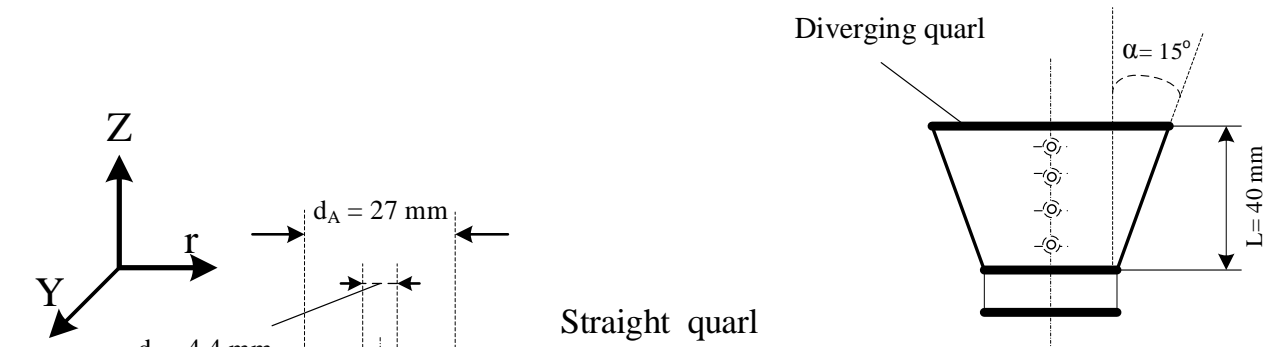

(b)

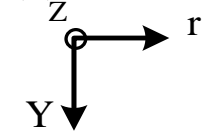

CMOS PIV

Throat $(Z=0)$

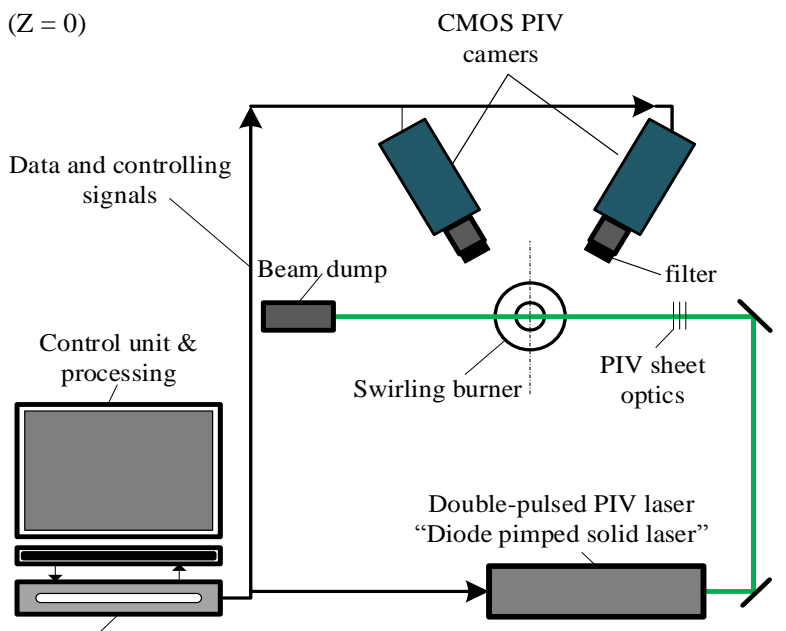

(c) (a)

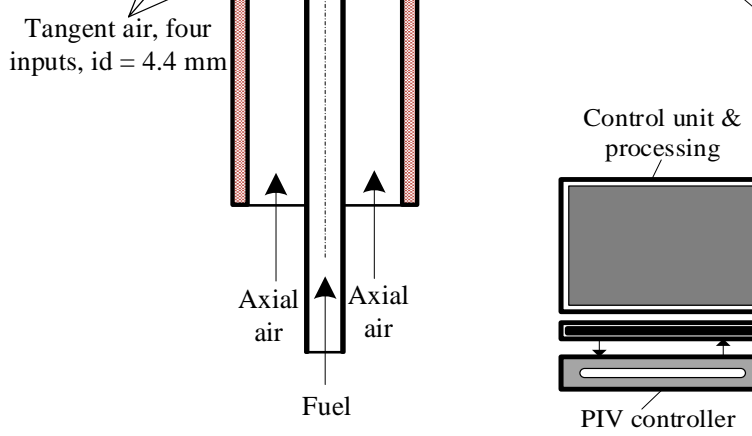

Fig. 1. Experimental set up, (a) swirling burner with straight quarl, (b) diverging quarl, (c) Stereo PIV set up 


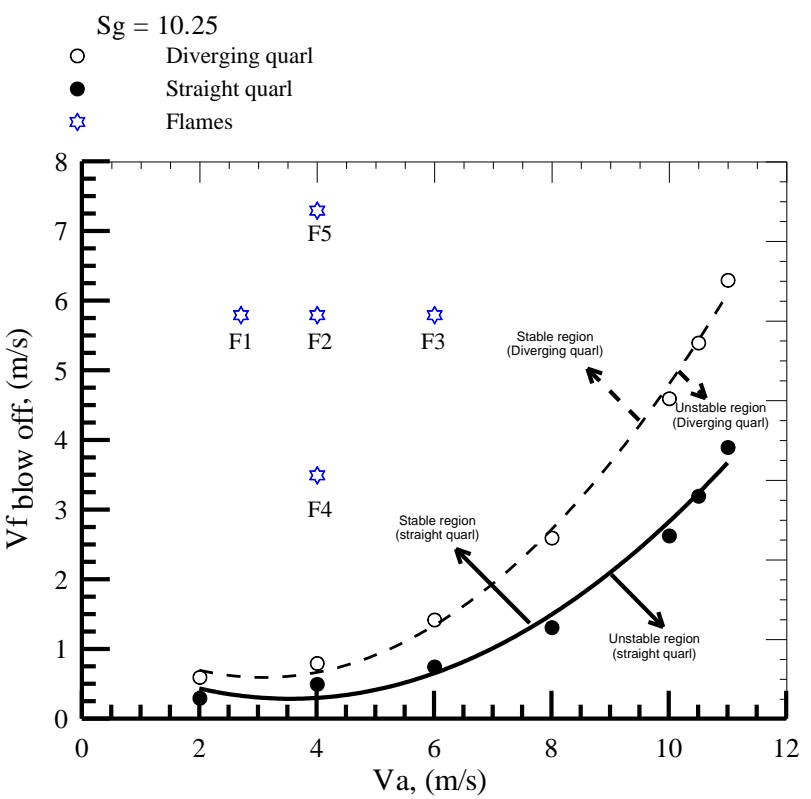

529

Fig. 2. Lean stability limits for straight and diverging quarl swirl stabilized methane non-premixed flame 531 at $(\mathrm{Sg}=10.25)$. The starts denote flames far from blow-off which are under investigation. 


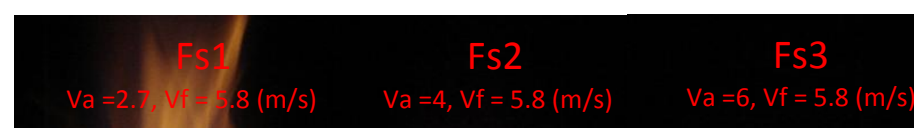

538

539

540

541

542

543

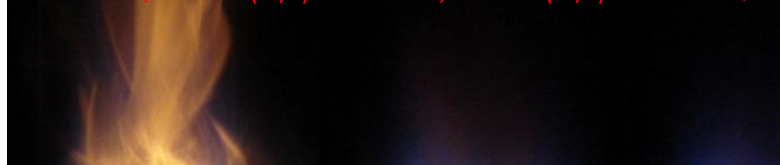

544

545

546

547

548

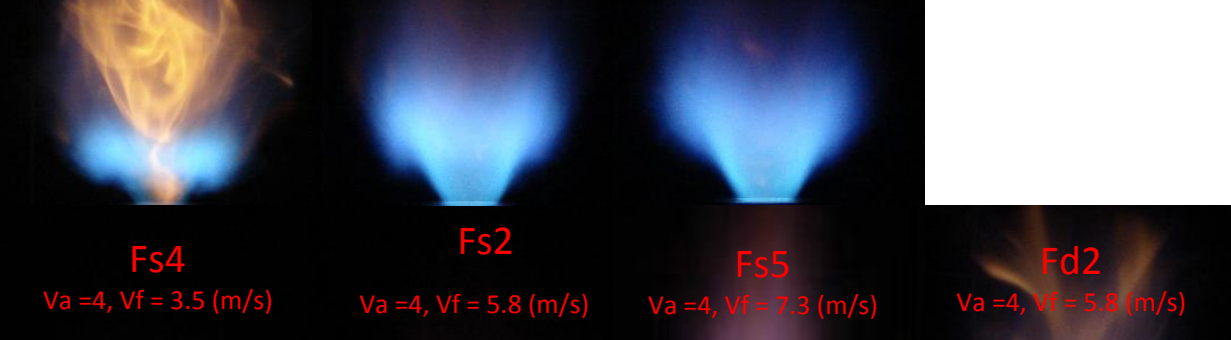

549 Fig. 3. Flames appearance, first raw show the effect of increasing the axial air velocity $(\mathrm{Sg}=10.25, \mathrm{Vf}=$ $5505.8 \mathrm{~m} / \mathrm{s}$ ), and in the second raw, from the first to the third photos indicate the effect of increasing fuel jet 551 velocity ( $\mathrm{Sg}=10.25, \mathrm{Va}=4 \mathrm{~m} / \mathrm{s}$, for clarity the photo of flame Fs2 is repeated), the last images "Fd2" 552 shows the effect of quarl angle on the flame appearance working the same flow conditions of flame Fs2. 

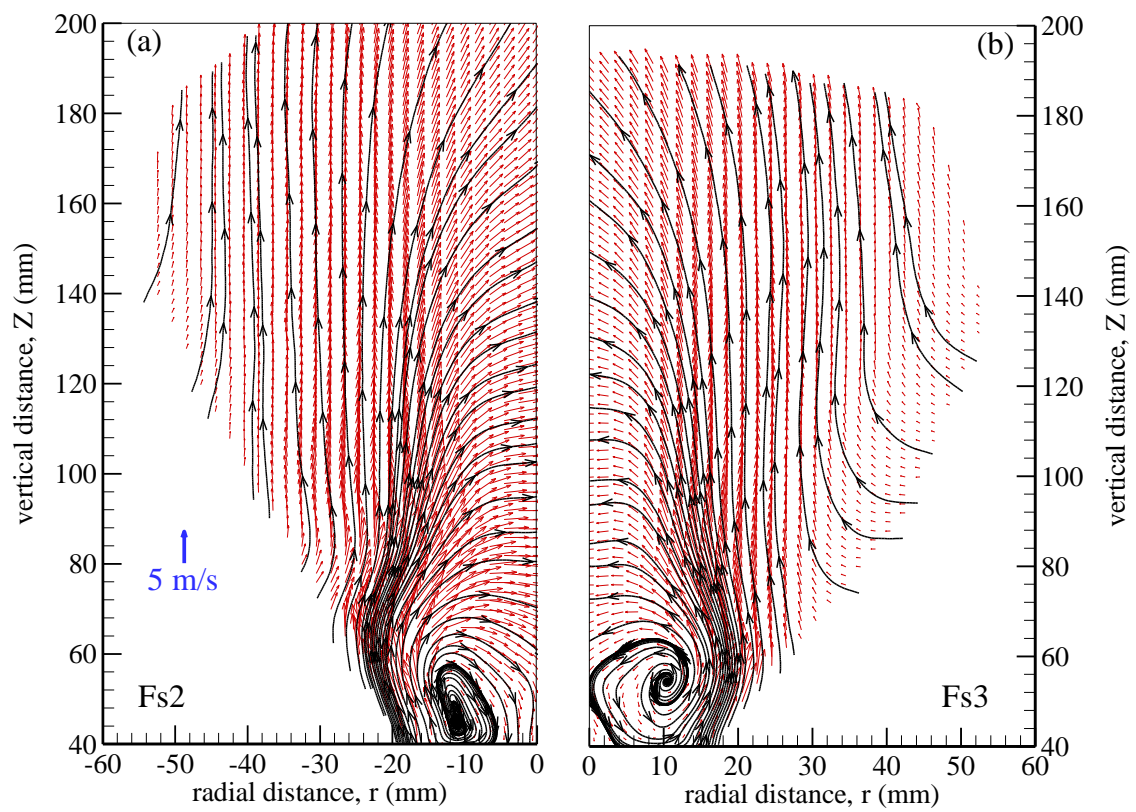

479

480

481
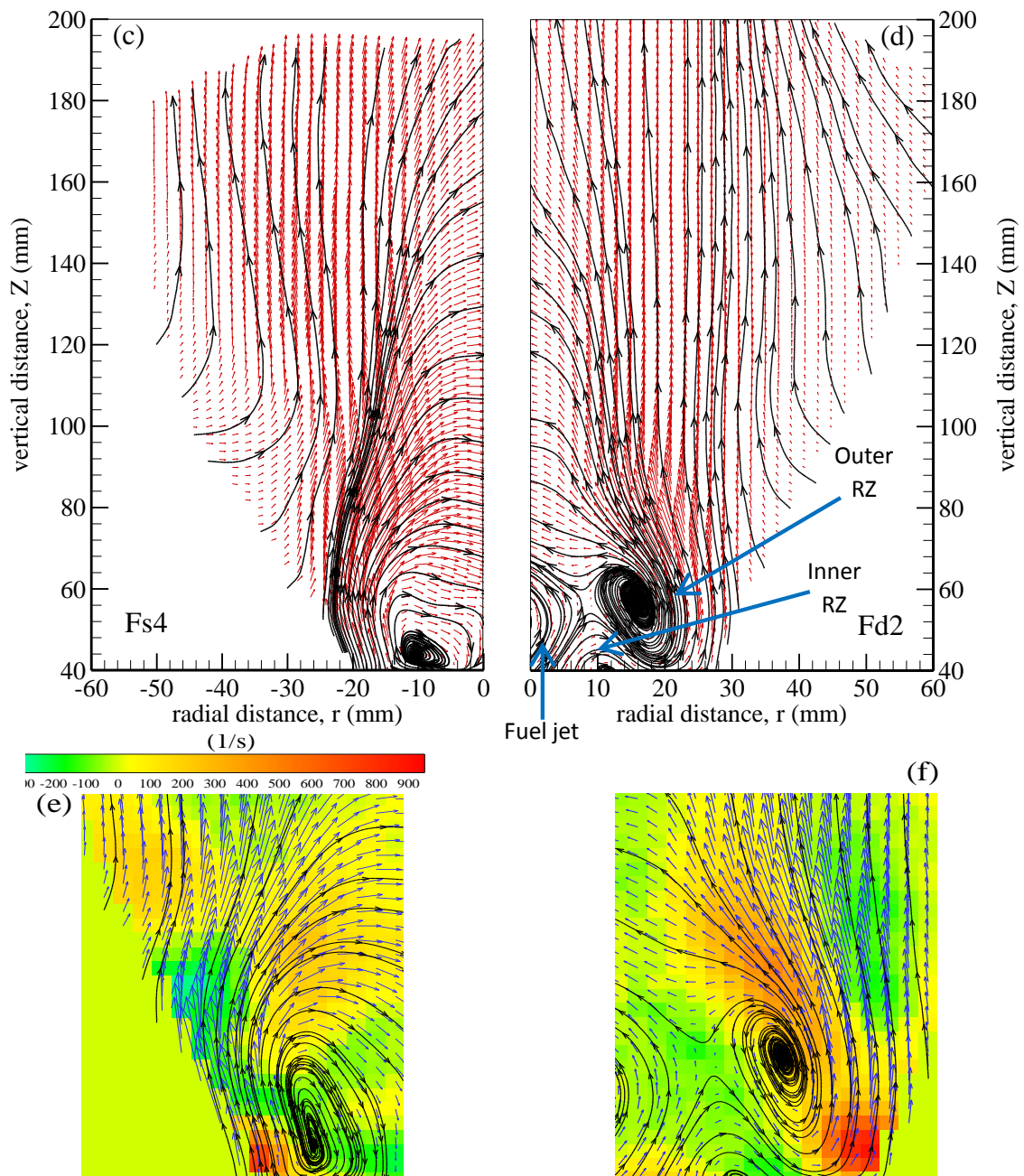

Fig. 4. Time average velocity vectors overlaid the averaged stream line plots at the axial plane of the burner: (a) flame Fs2, (b) flame Fs3, (c) flame Fs4, and (d) flame Fd2 (alternate rows of vectors have 499 been removed for clarity), (e) and (f) are the velocity vectors superimposed the average strain rate $(\partial \mathrm{v} / \partial \mathrm{z})$ of flames Fs2 and Fd2 respectively. 
(a) Fs2

500

501

502

503

504

505

506

507

508

509

510

511

512

513

514

515

516

517

518

519

520

521

522

523

524

525

526

527

528

529

(c)

(e)

8
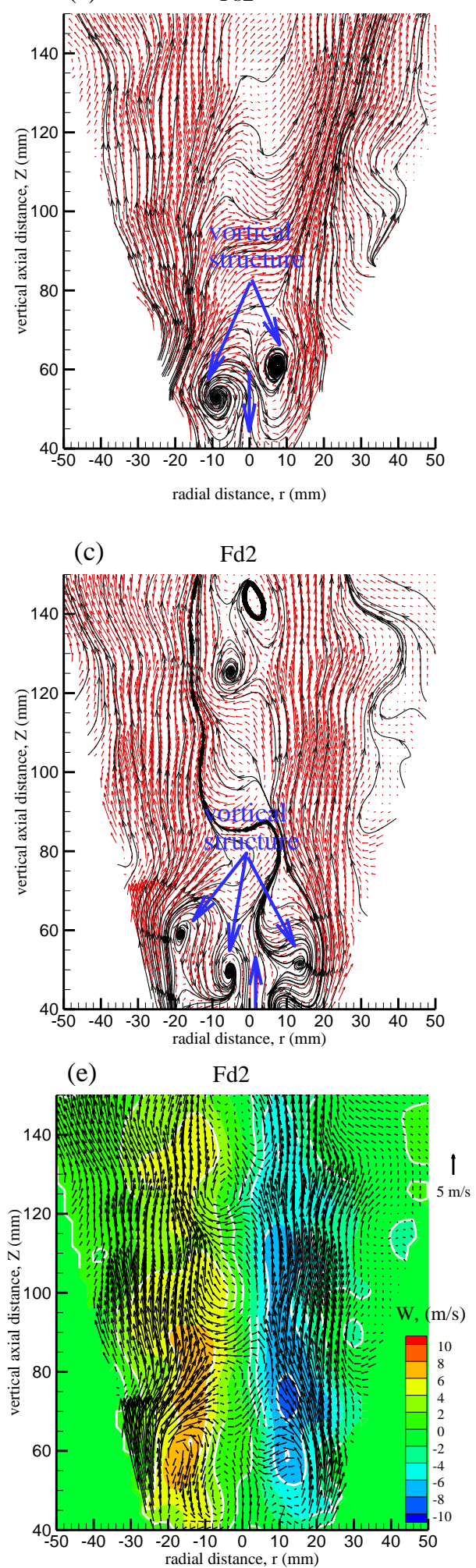

(b) Fs2

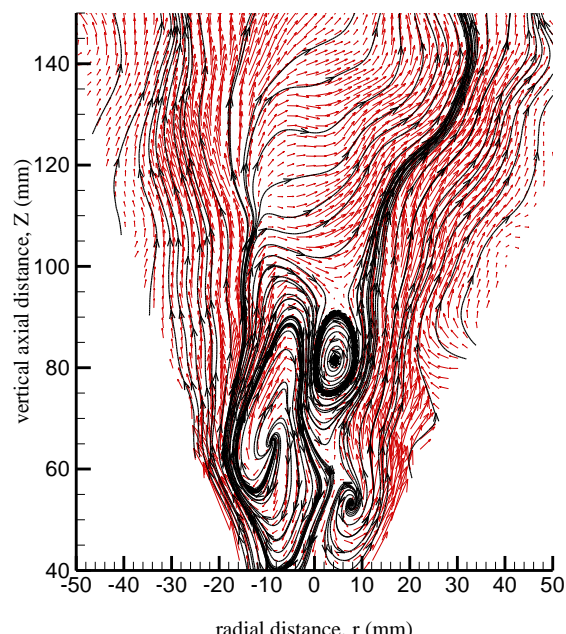

(d)

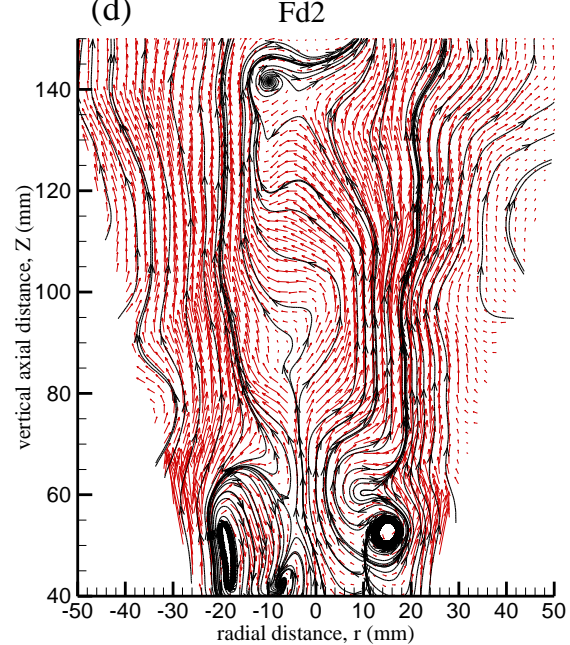

Fig. 5. Two examples of instantaneous velocity vectors and stream lines plot of the flow field at the axial plane: (a), (b) of flame Fs2, (c), (d) of Fd2, and (e) the velocity vector superimposed with the swirling velocity contours for Fd2 (alternate rows of vectors have been removed for clarity). 
531

532

533

534

535

536

537

538

539

540
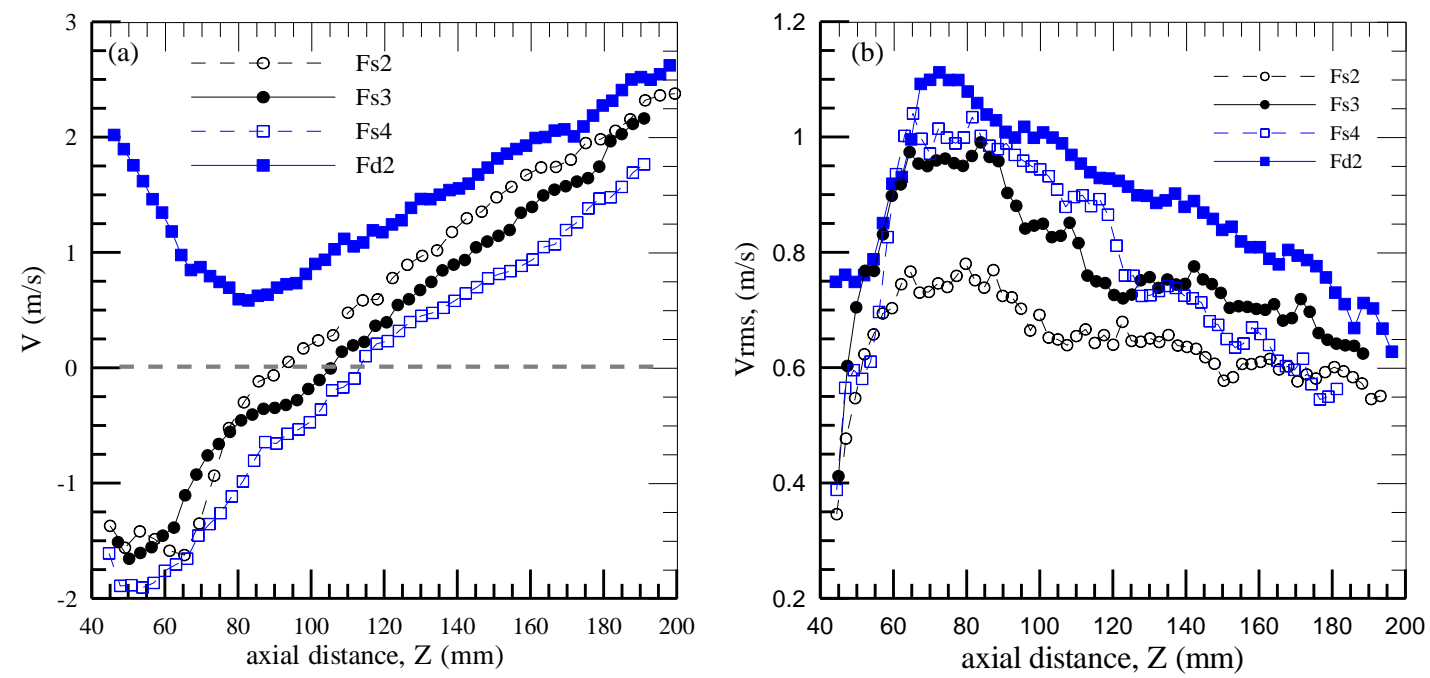

541

542

543

Fig. 6. Centerline profiles of (a) mean axial velocity, V, and (b) rms of the axial velocity, (Vrms) for flames Fs2, Fs3, Fs4, and Fd2

544

545 


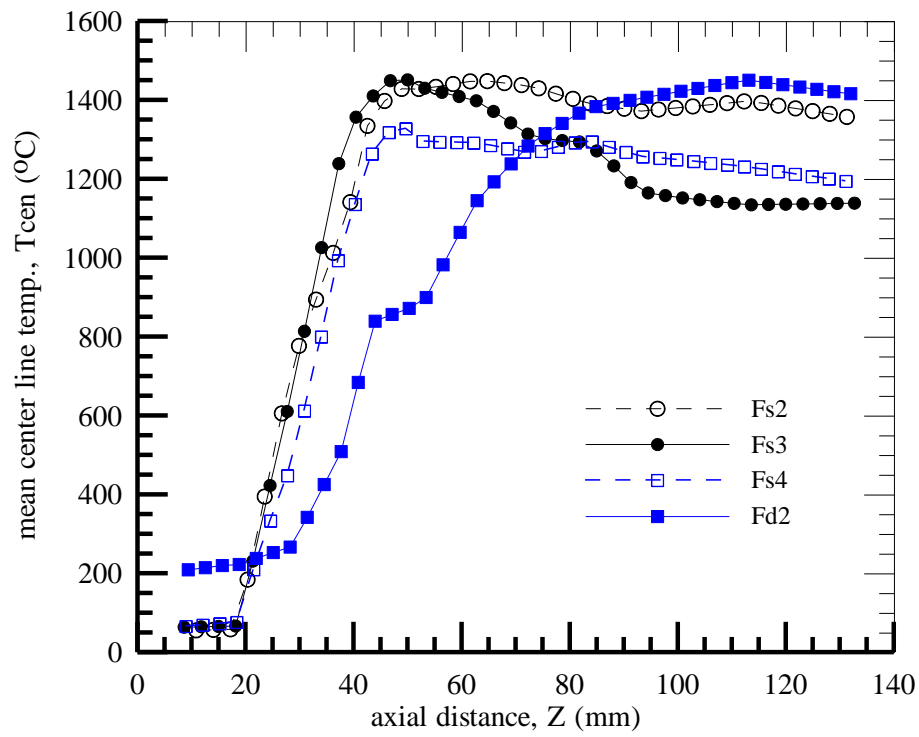

Fig. 7. Mean centerline temperature of flames, Fs2, Fs3, Fs4, and Fd2. 
561

562

563

564

565

566

567

568

569

570

571
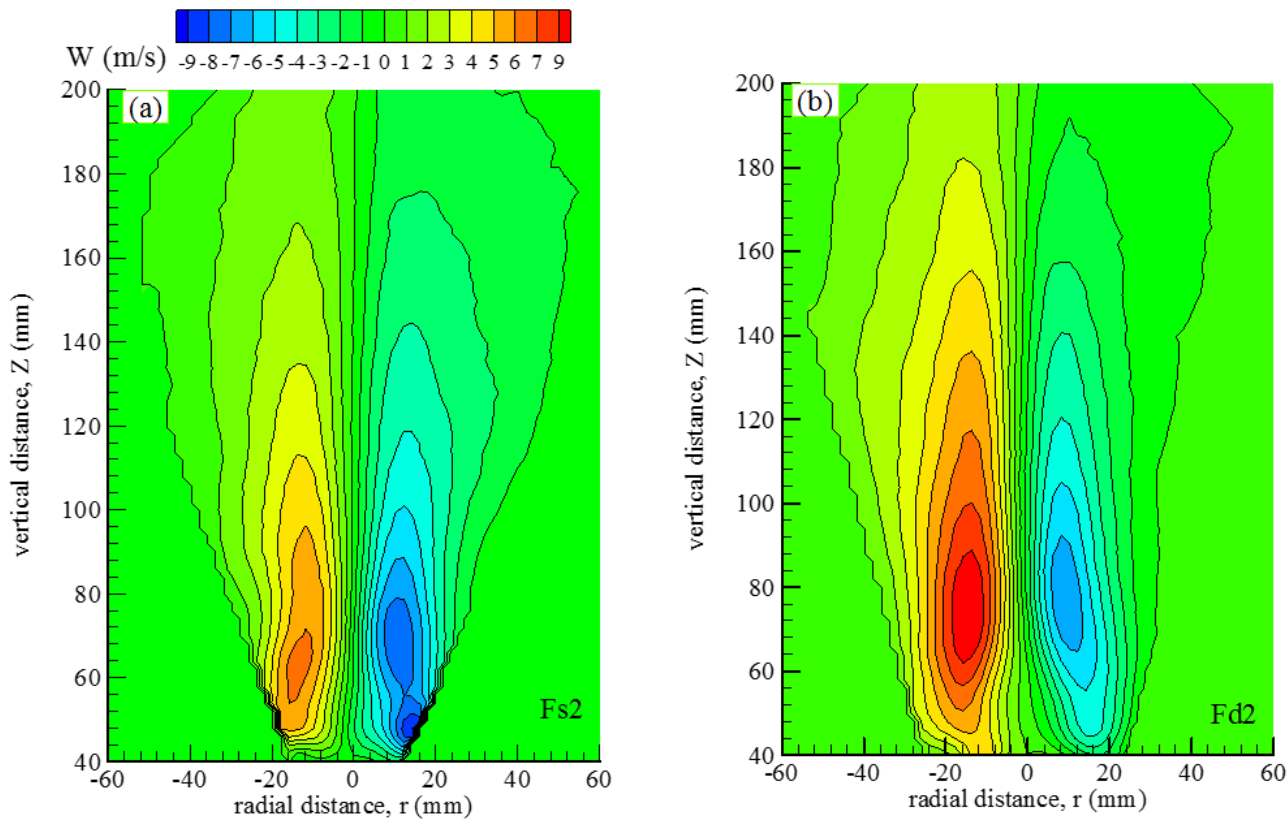

572

573 Fig. 8. Time average velocity contours of swirling velocity components of the flow field at the axial plane 574 of the burner: (a) Flame Fs2, (b) Flame Fd2.

575 


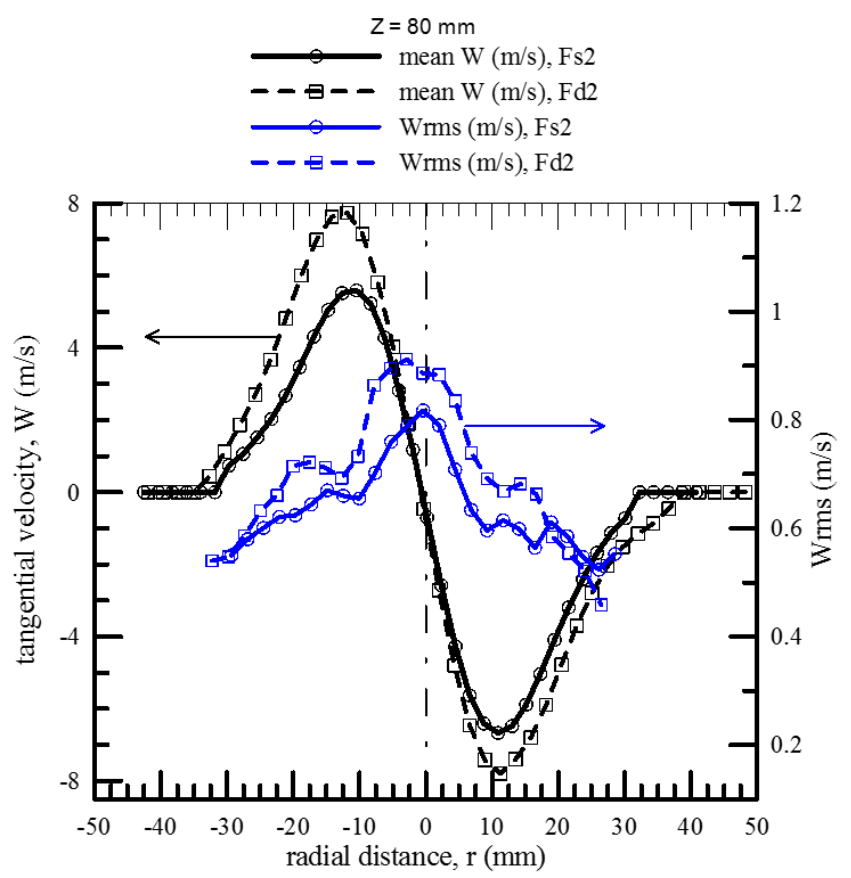

Fig. 9. Radial profiles of swirling velocity component, W, and Wrms, of flames Fs 2 and Fd2 at axial 591 
601

602

603

604

605
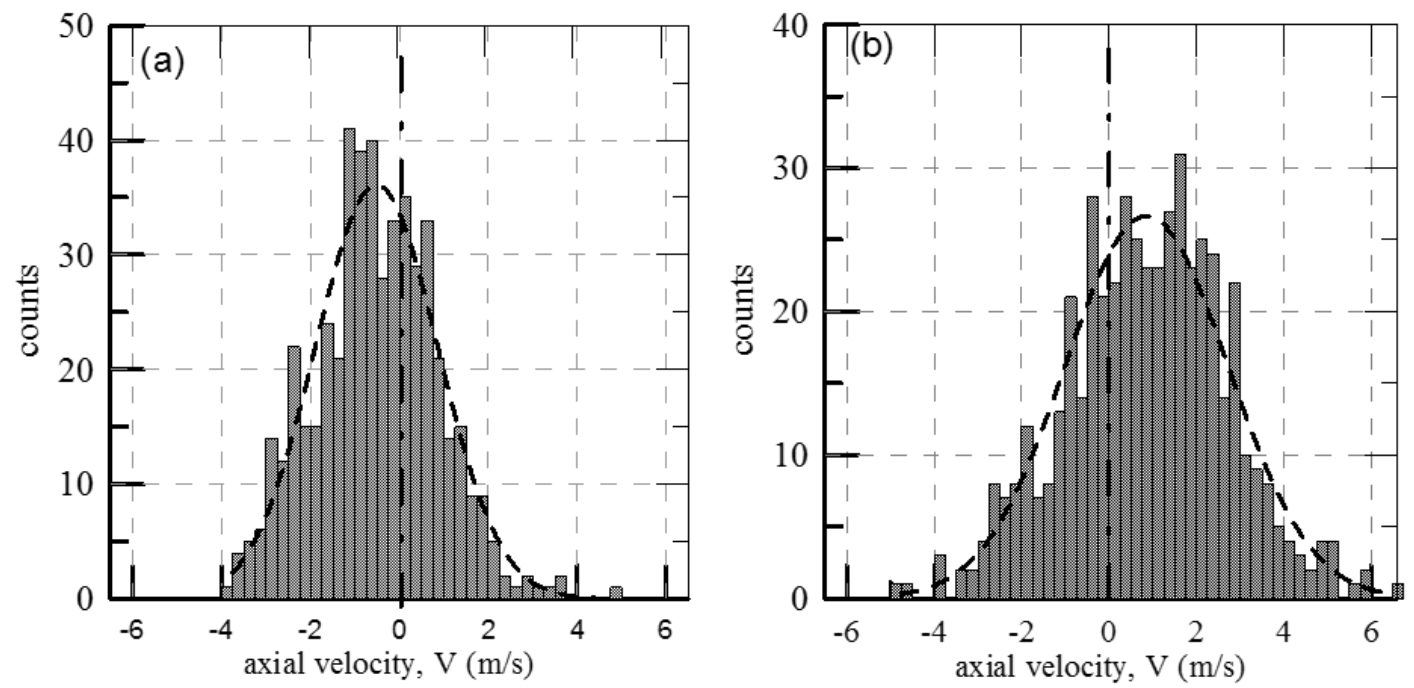

606

607

Fig. 10. Axial velocity histograms on the burner axis and at an axial location of $Z=60 \mathrm{~mm}$, (a) Flame Fs2 and (b) Flame Fd2.

608

609 
610

611

612

613

614

615

616

617

618

619

620

621

622

623

624

625

626

627

628

629

630

631

632

633

634

635

636

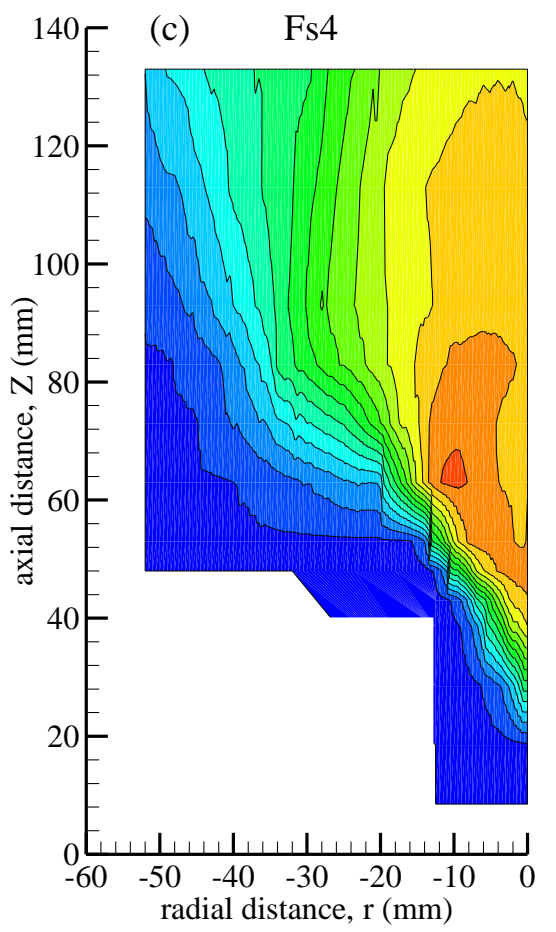

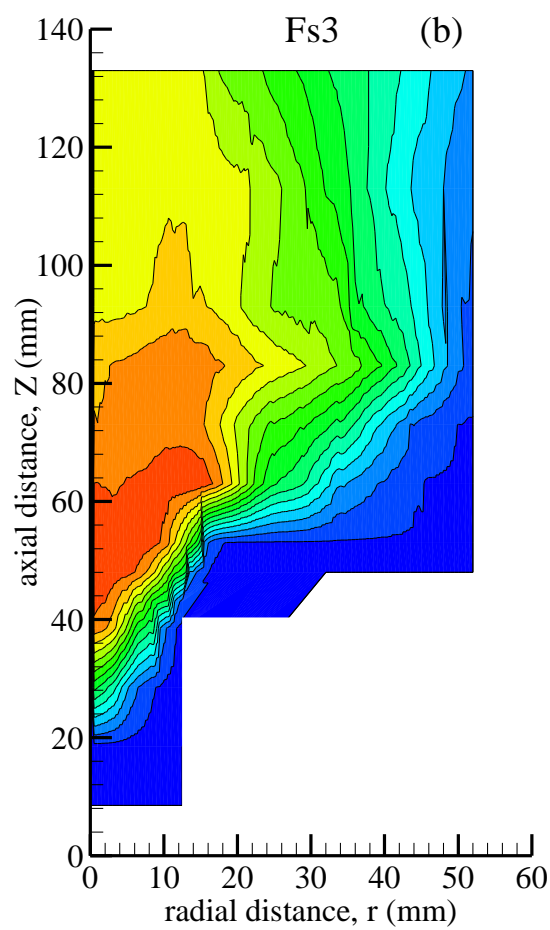

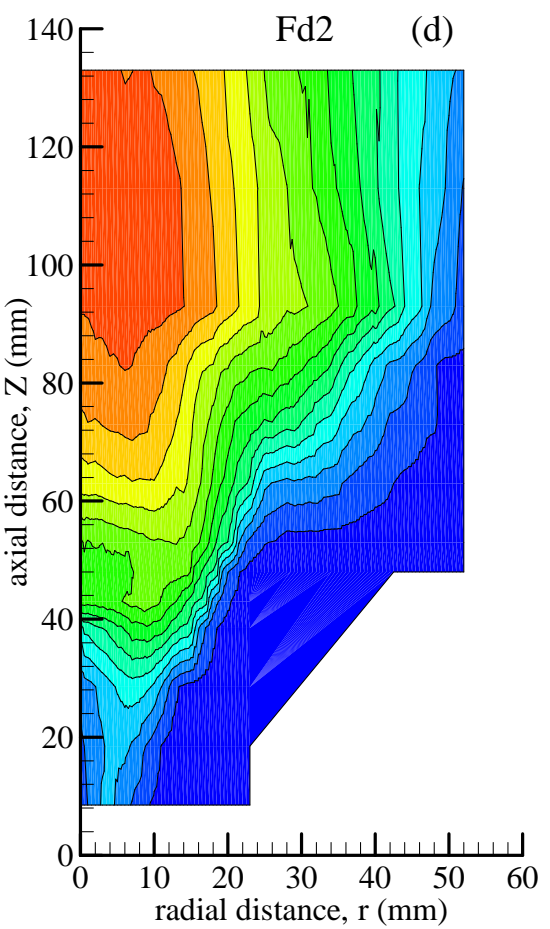

637

638 
644

645

646

647

648

649

650

651

652

653

654 (a)

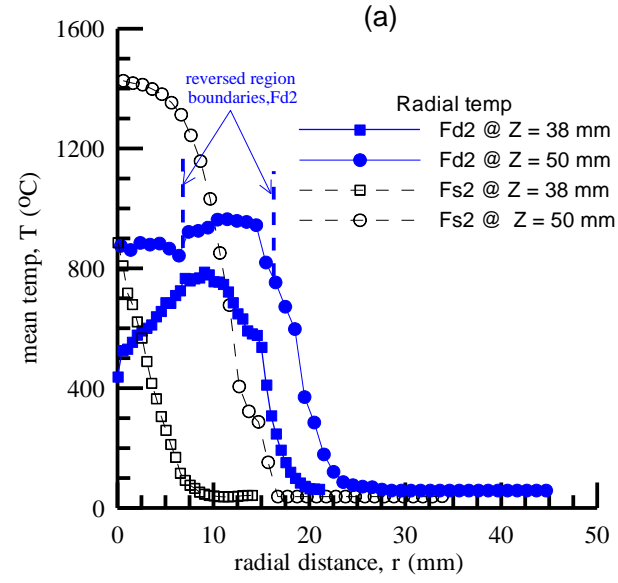

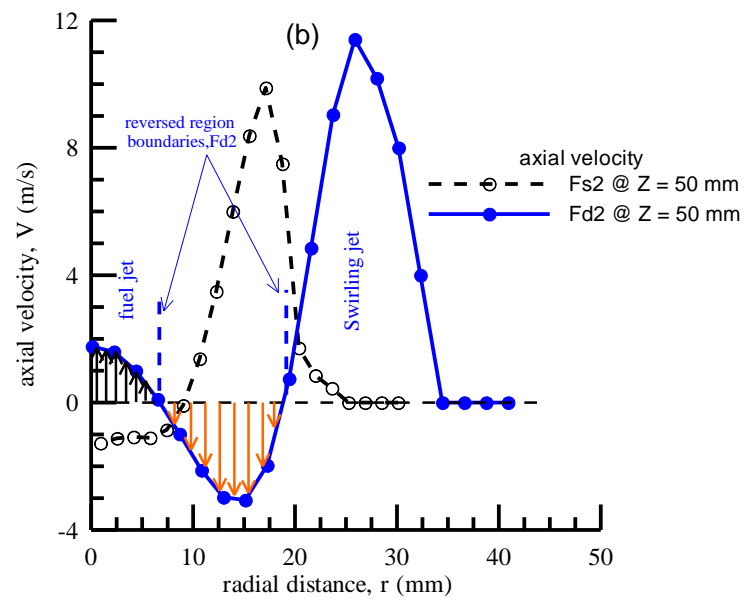

Fig. 12. Radial profiles, (a) temperature profile at axial distances at $Z=28$ and $50 \mathrm{~mm}$ of flames Fs 2 and $\mathrm{Fd} 2$, and (b) Radial axial velocity profiles at $\mathrm{Z}=50 \mathrm{~mm}$ of flames Fs2 and Fd2. 


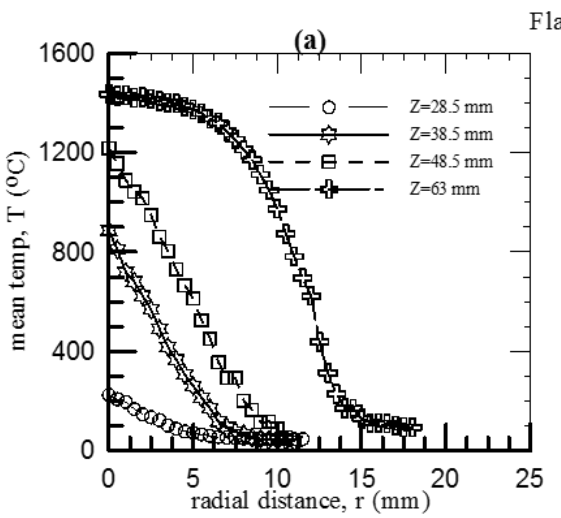

Flame Fs2
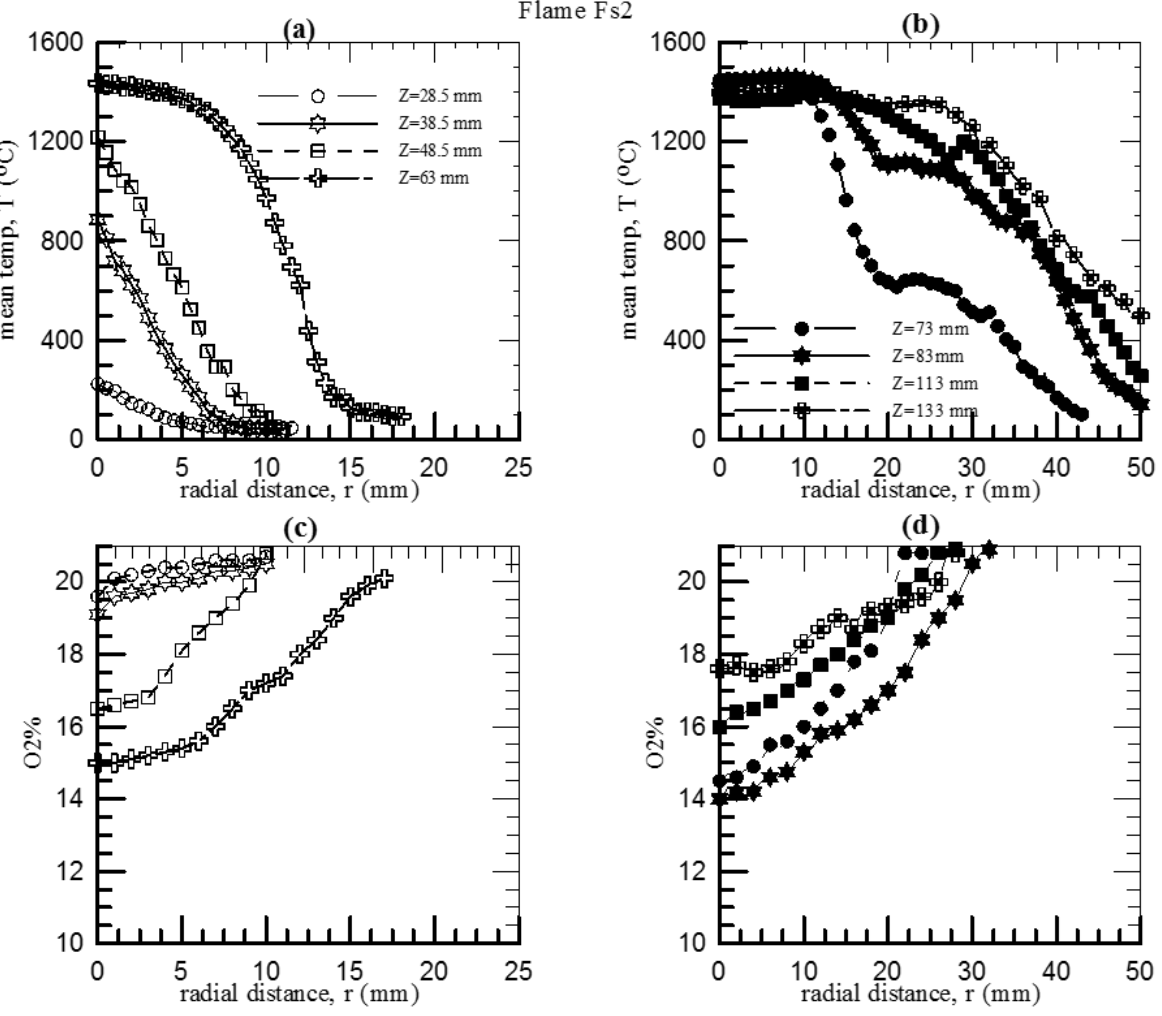

669

670

671

672

673

674

675

676

677

678

679

680

681
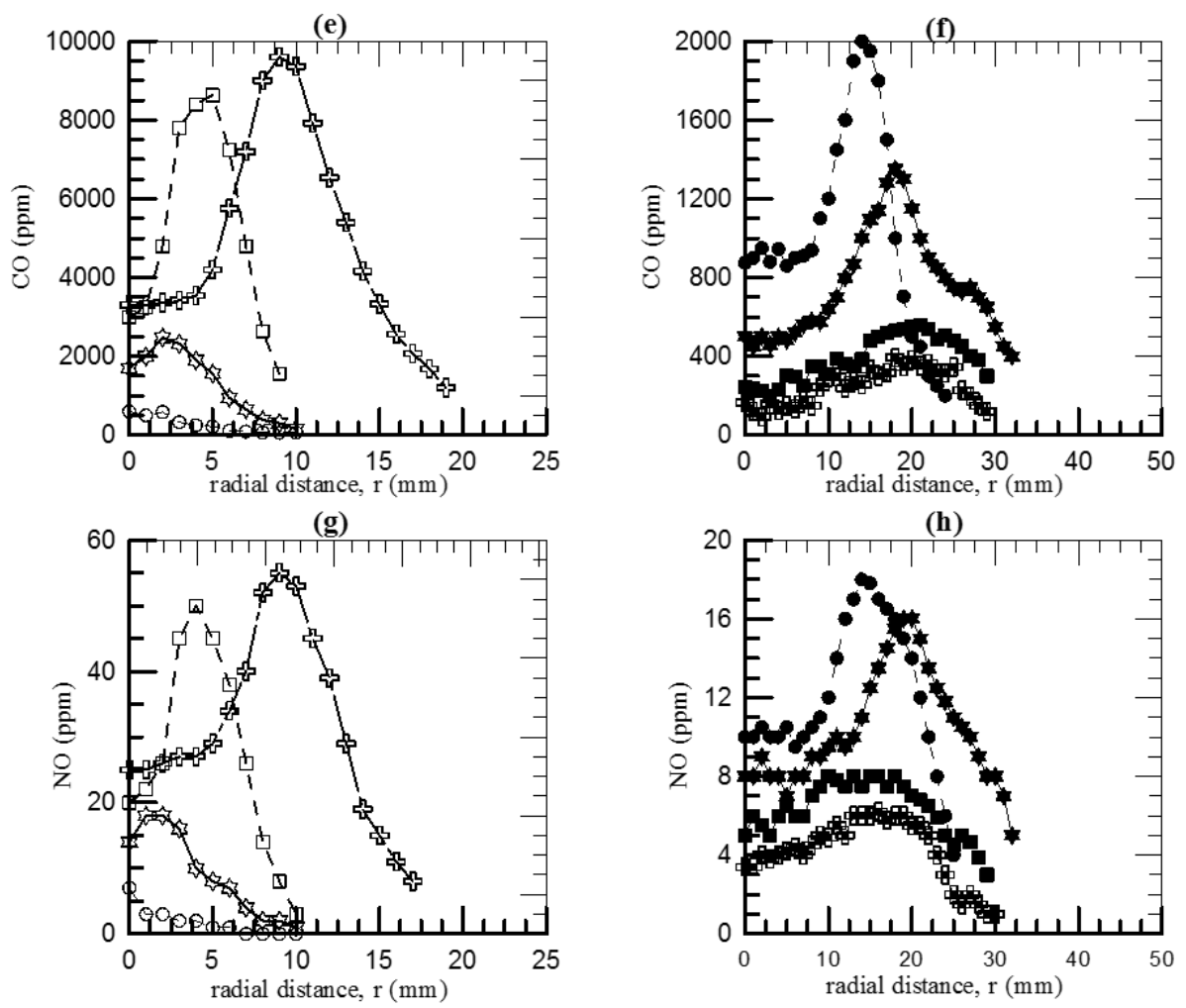

682

683

Fig. 13. Radial profiles of species concentration and mean temperature at different axial locations of flame Fs2. 


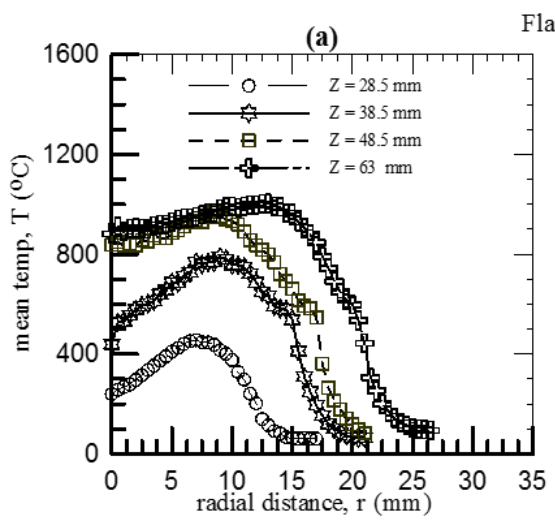

Flame Fd2
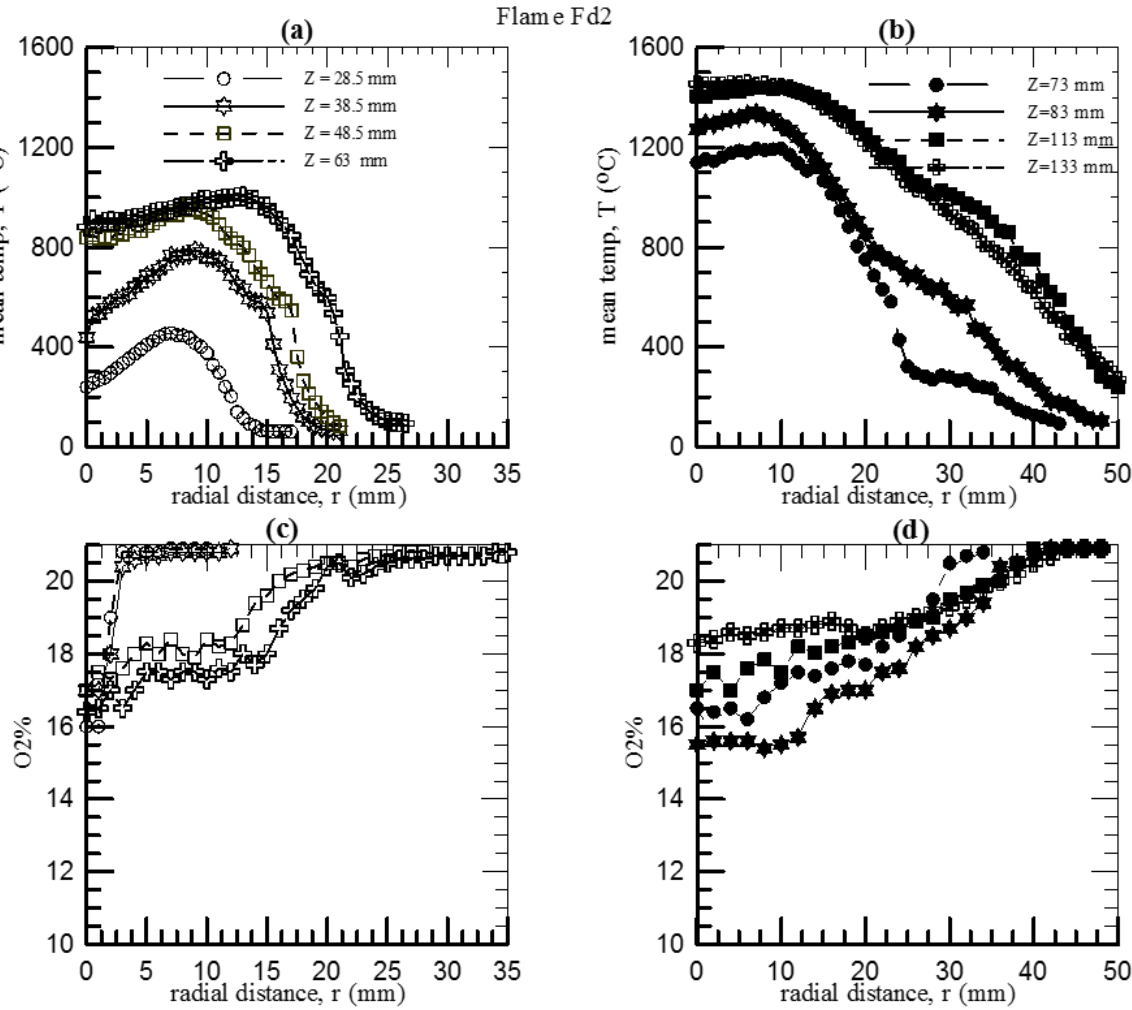

692

693

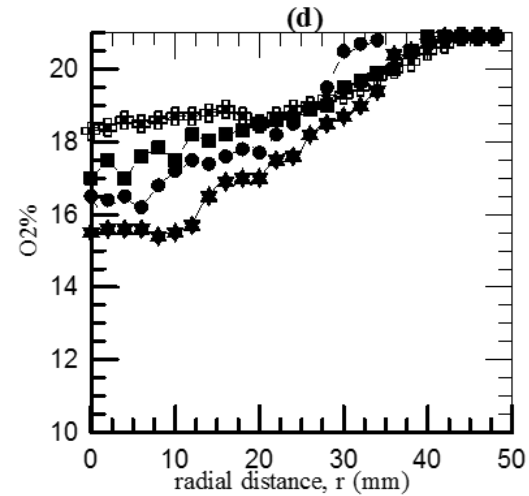

699

700

701

702

703

704
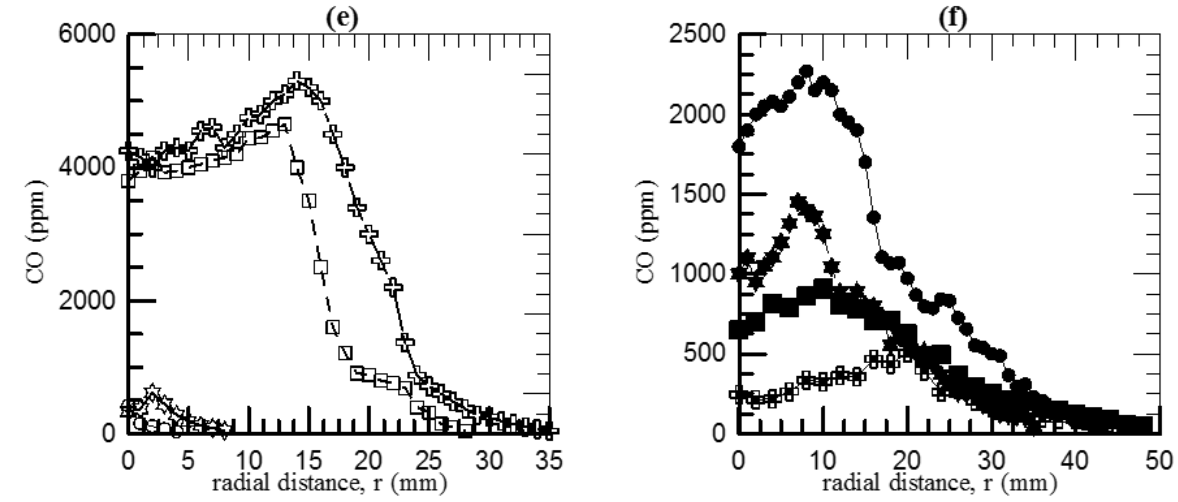

705

706

707

708

709
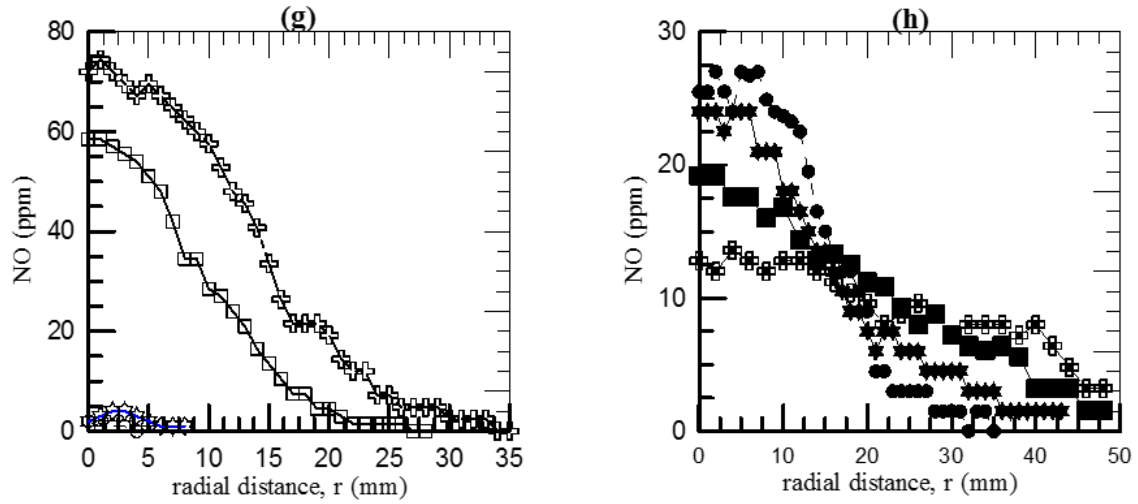

Fig. 14. Radial profiles of species concentration and mean temperature at different axial locations of flame $\mathrm{Fd} 2$. 